\title{
Global existence of weak solution and regularity criteria for the 2D Bénard system with partial dissipation
}

Liangliang $\mathrm{Ma}^{1}$ and Lei Zhang ${ }^{2,3,4^{*}}$

${ }^{\text {*Correspondence: }}$
zhanglei@scu.edu.cn
${ }^{2}$ College of Computer Science,
Sichuan University, Chengdu, P.R.
China
${ }^{3}$ State Key Laboratory of Plateau
Ecology and Agriculture,
Department of Computer
Technology and Applications,
Qinghai University, Xining, P.R. China
Full list of author information is
available at the end of the article

\section{Correspondence:} China

${ }^{3}$ State Key Laboratory of Plateau Ecology and Agriculture,

Technology and Applications,

Full list of author information is

available at the end of the article

\begin{abstract}
In this paper, we first write the velocity equation of the Bénard system in its two components, and consider the global weak solution of the resulting 2D Bénard system with partial dissipation, i.e. (1) $\mu_{1}=0, \mu_{2}>0, \mu_{3}=0, \mu_{4}=0, \kappa_{1}>0, \kappa_{2}=0$; (2) $\mu_{1}=0, \mu_{2}=0, \mu_{3}>0, \mu_{4}=0, \kappa_{1}=0, \kappa_{2}>0$; (3) $\mu_{1}>0, \mu_{2}=0, \mu_{3}=0, \mu_{4}=0$, $\kappa_{1}>0, \kappa_{2}=0$; (4) $\mu_{1}>0, \mu_{2}=0, \mu_{3}=0, \mu_{4}=0, \kappa_{1}=0, \kappa_{2}>0$; (5) $\mu_{1}=0, \mu_{2}>0$, $\mu_{3}=0, \mu_{4}=0, \kappa_{1}=0, \kappa_{2}>0$; (6) $\mu_{1}=0, \mu_{2}=0, \mu_{3}>0, \mu_{4}=0, \kappa_{1}>0, \kappa_{2}=0$; (7) $\mu_{1}=0, \mu_{2}=0, \mu_{3}=0, \mu_{4}>0, \kappa_{1}>0, \kappa_{2}=0$; (8) $\mu_{1}=0, \mu_{2}=0, \mu_{3}=0, \mu_{4}>0$, $\kappa_{1}=0, \kappa_{2}>0$, where $\mu_{i}(i=1,2,3,4)$ and $\kappa_{j}(j=1,2)$ are the coefficients of dissipation and thermal diffusivity. Furthermore, we establish some regularity criteria for the corresponding system. This work follows the techniques in the paper of Cao and Wu (Adv. Math. 226:1803-1822, 2011).
\end{abstract}

MSC: 35Q35; 35B45; 35B65

Keywords: Bénard system; Weak solution; Regularity criteria; Partial dissipation; Thermal diffusivity

\section{Introduction}

The Bénard system described the Rayleigh-Bénard convective motion in a heated 2D inviscid incompressible fluid under thermal effects (see e.g. [2-7]). One of the most fundamental problems in fluid dynamics concerning the Bénard system is whether their classical solutions are global regularity for all time or they develop singularities. The Bénard system has been a center of attention to numerous analytical, experimental, and number investigations. The motion of the incompressible Bénard system in $\mathbb{R}^{2}$ is governed by

$$
\left\{\begin{array}{l}
\partial_{t} u+(u \cdot \nabla) u+\nabla \pi=\mu \Delta u+\theta e_{2} \\
\partial_{t} \theta+(u \cdot \nabla) \theta=\kappa \Delta \theta+u \cdot e_{2}, \\
\nabla \cdot u=0
\end{array}\right.
$$

where the unknown functions $u, \theta$ and $\pi$ denote the $2 \mathrm{D}$ velocity field, temperature and pressure, respectively. The constants $\mu$ and $\kappa$ are the coefficients of dissipation and thermal diffusivity. The forcing term $\theta e_{2}$ in the momentum equation describes the acting of the buoyancy force on fluid motion and $u \cdot e_{2}$ models the Rayleigh-Bénard convection

(c) The Author(s) 2018. This article is distributed under the terms of the Creative Commons Attribution 4.0 International License (http://creativecommons.org/licenses/by/4.0/), which permits unrestricted use, distribution, and reproduction in any medium, provided you give appropriate credit to the original author(s) and the source, provide a link to the Creative Commons license, and indicate if changes were made. 
in a heated inviscid fluid, and $e_{2}=(0,1)^{T}$. Physically, $(1.1)_{1}$ reflects the conservation of momentum, $(1.1)_{2}$ describes the thermal convection, and in $(1.1)_{3}, \nabla \cdot u=0$ shows the conservation of mass.

In the fluid dynamics area, the Bénard fluid problem is a very classical problem, which has an important significance in convective motion in a heated fluid, such as the case of planar stationary flows. The incompressible Bénard system have attracted the attention of many physicists and mathematicians due to its important physical background, rich phenomena, mathematical complexity and challenges. Neustupa and Siginer [8] proved the existence of a strong-weak solution $(u, \pi, \theta)$ of the steady Bénard problem in a $2 \mathrm{D}$ quadrangular cavity, heated/cooled on two opposite sides and thermally insulated on the other sides. The nonlinear Lyapunov stability of the conduction-diffusion solution of the rotating Bénard problem was studied in [9]. Anh and Son [10] studied the 2D Bénard problem in an arbitrary domain (bounded or unbounded) which satisfying the Poincaré inequality with nonhomogeneous boundary conditions and nonautonomous external force and heat source, and the existence of a weak solution to the problem was proved by using the Galerkin method, and showed that the existence of a unique minimal finite-dimensional pullback $D_{\sigma}$-attractor for the process associated to the problem. Wu and Xue [11] considered the Cauchy problem of the 2D inviscid Bénard system with fractional diffusivity, and showed that the system had a unique global solution $(u, \theta)$ such that $u \in C^{0,1}\left(\mathbf{R}_{+}, L^{2}\left(\mathbb{R}^{2}\right)\right)$, $\theta \in C\left(\mathbb{R}_{+}, L^{2} \cap B_{\infty, 1}^{1-\beta}\right) \cap L_{\text {loc }}^{2}\left(\mathbb{R}_{+}, H^{\frac{\beta}{2}}\right)$. 2D incompressible Bénard system with critical and supercritical dissipation $(0 \leq \alpha \leq 1)$ in the velocity was studied in [12]. Cheng and $\mathrm{Du}$ [13] considered the Cauchy problem of the 2D magnetic Bénard problem with mixed partial viscosity. More precisely, the global well-posedness of the 2D magnetic Bénard problem without thermal diffusivity and with vertical or horizontal magnetic diffusion was obtained. Moreover, the global regularity and some conditional regularity of strong solutions were obtained for the 2D magnetic Bénard problem with mixed partial viscosity. Zhou-Nakamura [14] studied a 2D magnetic Bénard problem with zero thermal conductivity, and showed a global well-posedness result by a well-known property of Hardy space and BMO. As it is demonstrated in reference $[15,16]$, we showed the global regularity for the two-and-half-dimensional magnetic Bénard system with zero thermal diffusivity by a well-known property of Hardy space and BMO; resorting to the method of the localin-time analysis, the global regularity for the two-and-half-dimensional magnetic Bénard system with zero thermal diffusivity and horizontal magnetic diffusion as well as vertical magnetic diffusion are also obtained. Moreover, we proved that, as the initial data satisfy $\left\|u_{0}\right\|_{H_{\mathbb{R}^{3}}^{1}}^{2}+\left\|b_{0}\right\|_{H_{\mathbb{R}^{3}}^{1}}^{2}+\left\|\theta_{0}\right\|_{H_{\mathbb{R}^{3}}^{1}}^{2} \leq \varepsilon$, where $\varepsilon$ is a suitably small positive number, then the 3D magnetic Bénard system with mixed partial dissipation, magnetic diffusion and thermal diffusivity admits global smooth solutions. Zhang and Tang [17] studied the global regularity for a special family of axisymmetric solutions to the 3D magnetic Bénard problem.

In particular, if $\theta=$ Const., then system (1.1) reduces to the classical Navier-Stokes system which describes the motion of incompressible viscous fluid flows and has been extensively studied by many authors; see [18-24] and the references therein. In addition to this, the reader is referred to [25-29] to find more results about the related fluid flow equations.

Recently, Regmi [30] established global weak solution for the 2D MHD system with partial dissipation and vertical diffusion. Cheng and Li [31] established the global weak solutions for the 2D Boussinesq system with mixed partial dissipation and thermal diffusivity. Chen concerned with the 2D system of the incompressible micropolar fluid flows 
with mixed partial viscosity and angular viscosity, and the global existence and uniqueness of smooth solution was showed in [32]. Yu considered the global regularity to the initial-boundary value problem of the 2D incompressible MHD system with mixed partial dissipation and magnetic diffusion in [33]. Fan et al. considered the global regularity for the 2D liquid crystal model with mixed partial viscosity and global Cauchy problem of 2D generalized MHD equations, respectively, in [34, 35].

Inspired by this work, we consider the following Bénard system in this paper:

$$
\left\{\begin{array}{l}
\partial_{t} u_{1}+(u \cdot \nabla) u_{1}+\partial_{x} \pi=\mu_{1} \partial_{x x} u_{1}+\mu_{2} \partial_{y y} u_{1} \\
\partial_{t} u_{2}+(u \cdot \nabla) u_{2}+\partial_{y} \pi=\mu_{3} \partial_{x x} u_{2}+\mu_{4} \partial_{y y} u_{2}+\theta \\
\partial_{t} \theta+(u \cdot \nabla) \theta=\kappa_{1} \partial_{x x} \theta+\kappa_{2} \partial_{y y} \theta+u_{2} \\
\partial_{x} u_{1}+\partial_{x} u_{2}=0
\end{array}\right.
$$

where $u=\left(u_{1}, u_{2}\right)$. System (1.2) is capable of modeling the motion of anisotropic fluids for which the diffusion properties in different directions are different. Additionally, (1.2) allows us to explore the smooth effects of various partial dissipations. Furthermore, we consider the 2D Bénard system (1.2) with partial dissipation in the following eight cases:

(i) $\mu_{1}=0, \mu_{2}>0, \mu_{3}=0, \mu_{4}=0, \kappa_{1}>0, \kappa_{2}=0$;

(ii) $\mu_{1}=0, \mu_{2}=0, \mu_{3}>0, \mu_{4}=0, \kappa_{1}=0, \kappa_{2}>0$;

(iii) $\mu_{1}>0, \mu_{2}=0, \mu_{3}=0, \mu_{4}=0, \kappa_{1}>0, \kappa_{2}=0$;

(iv) $\mu_{1}>0, \mu_{2}=0, \mu_{3}=0, \mu_{4}=0, \kappa_{1}=0, \kappa_{2}>0$;

(v) $\mu_{1}=0, \mu_{2}>0, \mu_{3}=0, \mu_{4}=0, \kappa_{1}=0, \kappa_{2}>0$;

(vi) $\mu_{1}=0, \mu_{2}=0, \mu_{3}>0, \mu_{4}=0, \kappa_{1}>0, \kappa_{2}=0$;

(vii) $\mu_{1}=0, \mu_{2}=0, \mu_{3}=0, \mu_{4}>0, \kappa_{1}>0, \kappa_{2}=0$;

(viii) $\mu_{1}=0, \mu_{2}=0, \mu_{3}=0, \mu_{4}>0, \kappa_{1}=0, \kappa_{2}>0$.

In this paper, we equip system (1.2) with the following initial data:

$$
\begin{aligned}
& u_{1}(x, y, 0)=u_{1}^{0}(x, y), \quad u_{2}(x, y, 0)=u_{2}^{0}(x, y), \\
& \theta(x, y, 0)=\theta^{0}(x, y), \quad(x, y) \in \mathbb{R}^{2} .
\end{aligned}
$$

The plan of this paper is as follows. Firstly, we give two very useful lemmas and establish the global weak solution for the 2D Bénard system with vertical dissipation in the first component of velocity field and horizontal thermal diffusivity in Sect. 2 . We shall give the global regularity criteria for weak solution of the 2D Bénard system with vertical dissipation in the first component of velocity field and horizontal thermal diffusivity in Sect. 3. In Sect. 4, we will give the global existence and regularity criteria of weak solution to Bénard system with other cases for partial viscosity and thermal diffusivity.

Notations. We introduce some notations which are used in this paper. For $1 \leq p \leq \infty$, $L^{p}=L^{p}\left(\mathbb{R}^{2}\right)$ denotes the usual Lebesgue space with the norm $\|\cdot\|_{L^{p}}$. The usual Sobolev space of order $n$ is defined by $H^{n}=\left\{f \in L^{2}\left(\mathbb{R}^{2}\right) \mid \nabla^{n} f \in L^{2}\right\}$ with the norm $\|f\|_{H^{n}}=\left(\|f\|_{L^{2}}^{2}+\right.$ $\left.\left\|\nabla^{n} f\right\|_{L^{2}}^{2}\right)^{\frac{1}{2}}$.

\section{Global weak solution for the Bénard system with vertical dissipation and horizontal thermal diffusivity}

In this section, we will establish the global weak solution for the 2D Bénard system with vertical dissipation in the first component of velocity field and horizontal thermal diffusivity. 


\subsection{Preliminaries}

In this subsection, we first provide the lemma that bounds a triple-product in terms of the Lebesgue norms of the functions and their directional derivatives; see for example $[1,36$, 37]. The following anisotropic Sobolev inequality will play very important roles in proving our main results.

Lemma 2.1 Assume that $f, g, h, \partial_{x} g, \partial_{y} h \in L^{2}\left(\mathbb{R}^{2}\right)$. Then there exists an absolute constant C such that

$$
\iint_{\mathbb{R}^{2}}|f g h| d x d y d z \leq C\|f\|_{L^{2}}\|g\|_{L^{2}}^{\frac{1}{2}}\left\|\partial_{x} g\right\|_{L^{2}}^{\frac{1}{2}}\|h\|_{L^{2}}^{\frac{1}{2}}\left\|\partial_{y} h\right\|_{L^{2}}^{\frac{1}{2}}
$$

The following simple fact on the boundedness of Riesz transforms will also be used.

Lemma 2.2 (see [1]) Letf be a divergence-free vector field such that $\nabla f \in L^{\gamma}$ for $\gamma \in(1, \infty)$. Then there exists a pure constant $C>0$ (independent of $\gamma$ ) such that

$$
\|\nabla f\|_{L^{\gamma}} \leq \frac{C \gamma^{2}}{\gamma-1}\|\nabla \times f\|_{L^{\gamma}}
$$

For simplicity, throughout this paper, we use the same letter $C$ to denote various generic positive constants whose exact values are unimportant and may vary from line to line.

\subsection{Global weak solution}

Theorem 2.3 Let $\mu_{1}=0, \mu_{2}>0, \mu_{3}=0, \mu_{4}=0, \kappa_{1}>0, \kappa_{2}=0$. Suppose that $u_{1}^{0}, u_{2}^{0}, \theta^{0} \in$ $H^{1}\left(\mathbb{R}^{2}\right)$ and $\partial_{x} u_{1}^{0}+\partial_{y} u_{2}^{0}=0$. Then the problem (1.2)-(1.3) admits a global weak solution $\left(u_{1}, u_{2}, \theta\right)$, which obeys

$$
u_{1}, u_{2}, \theta \in L^{\infty}\left([0, T) ; H^{1}\left(\mathbb{R}^{2}\right)\right), \quad \partial_{y} u_{1}, \partial_{x} \theta \in L^{2}\left([0, T) ; H^{1}\left(\mathbb{R}^{2}\right)\right)
$$

for any $T>0$.

Theorem 2.3 follows from the following two lemmas immediately.

Lemma 2.4 Consider (1.2) with $\mu_{1}=0, \mu_{2}>0, \mu_{3}=0, \mu_{4}=0, \kappa_{1}>0, \kappa_{2}=0$. Let $u_{1}^{0}, u_{2}^{0}, \theta^{0} \in$ $L^{2}\left(\mathbb{R}^{2}\right)$, then, for any $T>0$ and $0<t<T$, we have

$$
\left\|u_{1}\right\|_{L^{2}}^{2}+\left\|u_{2}\right\|_{L^{2}}^{2}+\|\theta\|_{L^{2}}^{2}+\mu_{2} \int_{0}^{t}\left\|\partial_{y} u_{1}(\tau)\right\|_{L^{2}}^{2} d \tau+\kappa_{1} \int_{0}^{t}\left\|\partial_{x} \theta(\tau)\right\|_{L^{2}}^{2} d \tau \leq C .
$$

Proof Taking the $L^{2}$-inner product of the first three equations in (1.2) with $u_{1}, u_{2}$ and $\theta$, respectively, integrating the resulting equations by parts over $\mathbb{R}^{2}$, and using the divergencefree condition $\partial_{x} u_{1}+\partial_{x} u_{2}=0$, we find after adding them together that

$$
\begin{aligned}
& \frac{1}{2} \frac{d}{d t}\left(\left\|u_{1}(t)\right\|_{L^{2}}^{2}+\left\|u_{2}(t)\right\|_{L^{2}}^{2}+\|\theta(t)\|_{L^{2}}^{2}\right)+\mu_{2}\left\|\partial_{y} u_{1}\right\|_{L^{2}}^{2}+\kappa_{1}\left\|\partial_{x} \theta\right\|_{L^{2}}^{2} \\
& \quad=2 \iint_{\mathbb{R}^{2}} u_{2} \theta d x d y \\
& \quad \leq C\left(\left\|u_{2}\right\|_{L^{2}}^{2}+\|\theta\|_{L^{2}}^{2}\right) .
\end{aligned}
$$


This, together with the Gronwall's inequality, gives the desired estimates of (2.3), which implies

$$
\left\|u_{1}, u_{2}, \theta\right\|_{L^{\infty}\left([0, T) ; L^{2}\left(\mathbb{R}^{2}\right)\right)}+\mu_{2}\left\|\partial_{y} u_{1}\right\|_{L^{2}\left([0, T) ; L^{2}\left(\mathbb{R}^{2}\right)\right)}+\kappa_{1}\left\|\partial_{x} \theta\right\|_{L^{2}\left([0, T) ; L^{2}\left(\mathbb{R}^{2}\right)\right)} \leq C .
$$

We thus complete the proof of Lemma 2.4.

To obtain the $H^{1}$-estimates for $u_{1}, u_{2}$ and $\theta$, we consider the following equations:

$$
\left\{\begin{array}{l}
\partial_{t} \omega+(u \cdot \nabla) \omega=\mu_{2} \partial_{y y y} u_{1}+\partial_{x} \theta, \\
\partial_{t} \partial_{x} \theta+\partial_{x}[(u \cdot \nabla) \theta]=\kappa_{1} \partial_{x x x} \theta+\partial_{x} u_{2}, \\
\partial_{t} \partial_{y} \theta+\partial_{y}[(u \cdot \nabla) \theta]=\kappa_{1} \partial_{x x y} \theta+\partial_{y} u_{2},
\end{array}\right.
$$

where the vorticity is $\omega=\partial_{x} u_{2}-\partial_{y} u_{1}$.

Lemma 2.5 Consider (1.2) with $\mu_{1}=0, \mu_{2}>0, \mu_{3}=0, \mu_{4}=0, \kappa_{1}>0, \kappa_{2}=0$. Let $u_{1}^{0}, u_{2}^{0}, \theta^{0} \in$ $H^{1}\left(\mathbb{R}^{2}\right)$, then, for any $T>0$ and $0<t<T$, we have

$$
\begin{gathered}
\|\omega\|_{L^{2}}^{2}+\left\|\partial_{x} \theta\right\|_{L^{2}}^{2}+\left\|\partial_{y} \theta\right\|_{L^{2}}^{2}+\mu_{2} \int_{0}^{t}\left\|\partial_{x y} u_{1}(\tau)\right\|_{L^{2}}^{2} d \tau+\mu_{2} \int_{0}^{t}\left\|\partial_{y y} u_{1}(\tau)\right\|_{L^{2}}^{2} d \tau \\
+\kappa_{1} \int_{0}^{t}\left\|\partial_{x x} \theta(\tau)\right\|_{L^{2}}^{2} d \tau+\kappa_{1} \int_{0}^{t}\left\|\partial_{x y} \theta(\tau)\right\|_{L^{2}}^{2} d \tau \leq C .
\end{gathered}
$$

Proof Multiplying the first equation of (2.6) by $\omega$ and integrating it over $\mathbb{R}^{2}$, we deduce

$$
\begin{aligned}
& \frac{1}{2} \frac{d}{d t}\|\omega(t)\|_{L^{2}}^{2}+\mu_{2}\left\|\partial_{x y} u_{1}\right\|_{L^{2}}^{2}+\mu_{2}\left\|\partial_{y y} u_{1}\right\|_{L^{2}}^{2} \\
& \quad=\iint_{\mathbb{R}^{2}} \partial_{x} \theta \omega d x d y \\
& \quad \leq C\left(\left\|\partial_{x} \theta\right\|_{L^{2}}^{2}+\|\omega\|_{L^{2}}^{2}\right),
\end{aligned}
$$

and hence, using (2.3), (2.5) and Gronwall's inequality, we know that

$$
\|\omega\|_{L^{2}}^{2}+\mu_{2} \int_{0}^{t}\left\|\partial_{x y} u_{1}(\tau)\right\|_{L^{2}}^{2} d \tau+\mu_{2} \int_{0}^{t}\left\|\partial_{y y} u_{1}(\tau)\right\|_{L^{2}}^{2} d \tau \leq C
$$

which gives

$$
\|\omega\|_{L^{\infty}\left([0, T) ; L^{2}\left(\mathbb{R}^{2}\right)\right)}+\mu_{2}\left\|\nabla \partial_{y} u_{1}\right\|_{L^{2}\left([0, T) ; L^{2}\left(\mathbb{R}^{2}\right)\right)} \leq C .
$$

On the other hand, multiplying $(2.6)_{2}$ and $(2.6)_{3}$, respectively, by $\partial_{x} \theta$ and $\partial_{y} \theta$, and integrating by parts, yields

$$
\frac{1}{2} \frac{d}{d t}\left(\left\|\partial_{x} \theta(t)\right\|_{L^{2}}^{2}+\left\|\partial_{y} \theta(t)\right\|_{L^{2}}^{2}\right)+\kappa_{1}\left\|\partial_{x x} \theta\right\|_{L^{2}}^{2}+\kappa_{1}\left\|\partial_{x y} \theta\right\|_{L^{2}}^{2}=I=\sum_{l=1}^{4} I_{l},
$$


where

$$
\begin{array}{ll}
I_{1}=-\iint_{\mathbb{R}^{2}} \partial_{x}[(u \cdot \nabla) \theta] \partial_{x} \theta d x d y, & I_{2}=\iint_{\mathbb{R}^{2}} \partial_{x} u_{2} \partial_{x} \theta d x d y, \\
I_{3}=-\iint_{\mathbb{R}^{2}} \partial_{y}[(u \cdot \nabla) \theta] \partial_{y} \theta d x d y, & I_{4}=\iint_{\mathbb{R}^{2}} \partial_{y} u_{2} \partial_{y} \theta d x d y .
\end{array}
$$

We now estimate the right-hand side of (2.11) term by term. We first write $I_{1}$ as

$$
\begin{aligned}
I_{1} & =-\iint_{\mathbb{R}^{2}} \partial_{x}[(u \cdot \nabla) \theta] \partial_{x} \theta d x d y \\
& =-\iint_{\mathbb{R}^{2}} \partial_{x} u_{1} \partial_{x} \theta \partial_{x} \theta d x d y-\iint_{\mathbb{R}^{2}} \partial_{x} u_{2} \partial_{y} \theta \partial_{x} \theta d x d y \\
& =I_{11}+I_{12} .
\end{aligned}
$$

Here, we have used the fact $\iint_{\mathbb{R}^{2}} u_{1} \partial_{x x} \theta \partial_{x} \theta d x d y+\iint_{\mathbb{R}^{2}} u_{2} \partial_{x y} \theta \partial_{x} \theta d x d y=0$, which can easily be obtained by integration by parts and the incompressible condition.

With the help of Lemma 2.1, the Cauchy-Schwarz inequality and Young's inequality, we infer that

$$
\begin{aligned}
I_{11} & =-\iint_{\mathbb{R}^{2}} \partial_{x} u_{1} \partial_{x} \theta \partial_{x} \theta d x d y \\
& \leq C\left\|\partial_{x} u_{1}\right\|_{L^{2}}\left\|\partial_{x} \theta\right\|_{L^{2}}^{\frac{1}{2}}\left\|\partial_{x x} \theta\right\|_{L^{2}}^{\frac{1}{2}}\left\|\partial_{x} \theta\right\|_{L^{2}}^{\frac{1}{2}}\left\|\partial_{x y} \theta\right\|_{L^{2}}^{\frac{1}{2}} \\
& \leq \frac{\kappa_{1}}{2}\left\|\partial_{x x} \theta\right\|_{L^{2}}^{2}+\frac{\kappa_{1}}{8}\left\|\partial_{x y} \theta\right\|_{L^{2}}^{2}+C\|\omega\|_{L^{2}}^{2}\left\|\partial_{x} \theta\right\|_{L^{2}}^{2} .
\end{aligned}
$$

Similarly, we obtain

$$
\begin{aligned}
I_{12} & =-\iint_{\mathbb{R}^{2}} \partial_{x} u_{2} \partial_{y} \theta \partial_{x} \theta d x d y \\
& \leq C\left\|\partial_{x} u_{2}\right\|_{L^{2}}\left\|\partial_{y} \theta\right\|_{L^{2}}^{\frac{1}{2}}\left\|\partial_{x y} \theta\right\|_{L^{2}}^{\frac{1}{2}}\left\|\partial_{x} \theta\right\|_{L^{2}}^{\frac{1}{2}}\left\|\partial_{x y} \theta\right\|_{L^{2}}^{\frac{1}{2}} \\
& \leq \frac{\kappa_{1}}{8}\left\|\partial_{x y} \theta\right\|_{L^{2}}^{2}+C\|\omega\|_{L^{2}}^{2}\left(\left\|\partial_{x} \theta\right\|_{L^{2}}^{2}+\left\|\partial_{y} \theta\right\|_{L^{2}}^{2}\right) .
\end{aligned}
$$

$I_{3}$ can be bounded by

$$
\begin{aligned}
I_{3}= & -\iint_{\mathbb{R}^{2}} \partial_{y}[(u \cdot \nabla) \theta] \partial_{y} \theta d x d y \\
= & -\iint_{\mathbb{R}^{2}} \partial_{y}\left(u_{1} \partial_{x} \theta+u_{2} \partial_{y} \theta\right) \partial_{y} \theta d x d y \\
= & -\iint_{\mathbb{R}^{2}} \partial_{y} u_{1} \partial_{x} \theta \partial_{y} \theta d x d y-\iint_{\mathbb{R}^{2}} u_{1} \partial_{x y} \theta \partial_{y} \theta d x d y \\
& -\iint_{\mathbb{R}^{2}} \partial_{y} u_{2} \partial_{y} \theta \partial_{y} \theta d x d y-\iint_{\mathbb{R}^{2}} u_{2} \partial_{y y} \theta \partial_{y} \theta d x d y \\
= & -\iint_{\mathbb{R}^{2}} \partial_{y} u_{1} \partial_{x} \theta \partial_{y} \theta d x d y-\iint_{\mathbb{R}^{2}} \partial_{y} u_{2} \partial_{y} \theta \partial_{y} \theta d x d y \\
= & -\iint_{\mathbb{R}^{2}} \partial_{y} u_{1} \partial_{x} \theta \partial_{y} \theta d x d y-2 \iint_{\mathbb{R}^{2}} u_{1} \partial_{y} \theta \partial_{x y} \theta d x d y
\end{aligned}
$$




$$
\begin{aligned}
\leq & C\left\|\partial_{y} u_{1}\right\|_{L^{2}}\left\|\partial_{x} \theta\right\|_{L^{2}}^{\frac{1}{2}}\left\|\partial_{x y} \theta\right\|_{L^{2}}^{\frac{1}{2}}\left\|\partial_{y} \theta\right\|_{L^{2}}^{\frac{1}{2}}\left\|\partial_{x y} \theta\right\|_{L^{2}}^{\frac{1}{2}} \\
& +C\left\|\partial_{x y} \theta\right\|_{L^{2}}\left\|u_{1}\right\|_{L^{2}}^{\frac{1}{2}}\left\|\partial_{y} u_{1}\right\|_{L^{2}}^{\frac{1}{2}}\left\|\partial_{y} \theta\right\|_{L^{2}}^{\frac{1}{2}}\left\|\partial_{x y} \theta\right\|_{L^{2}}^{\frac{1}{2}} \\
\leq & \frac{\kappa_{1}}{4}\left\|\partial_{x y} \theta\right\|_{L^{2}}^{2}+C\left(\left\|\partial_{y} u_{1}\right\|_{L^{2}}^{2}+\left\|u_{1}\right\|_{L^{2}}^{2}\left\|\partial_{y} u_{1}\right\|_{L^{2}}^{2}\right)\left(\left\|\partial_{x} \theta\right\|_{L^{2}}^{2}+\left\|\partial_{y} \theta\right\|_{L^{2}}^{2}\right) .
\end{aligned}
$$

In what follows, we estimate $I_{2}$ and $I_{4}$. Using the Cauchy inequality, we have

$$
I_{2}=\iint_{\mathbb{R}^{2}} \partial_{x} u_{2} \partial_{x} \theta d x d y \leq C\left(\|\omega\|_{L^{2}}^{2}+\left\|\partial_{x} \theta\right\|_{L^{2}}^{2}\right)
$$

and

$$
I_{4}=\iint_{\mathbb{R}^{2}} \partial_{y} u_{2} \partial_{y} \theta d x d y \leq C\left(\|\omega\|_{L^{2}}^{2}+\left\|\partial_{y} \theta\right\|_{L^{2}}^{2}\right) .
$$

Combining the above estimates, we conclude that

$$
\begin{aligned}
& \frac{d}{d t}\left(\left\|\partial_{x} \theta(t)\right\|_{L^{2}}^{2}+\left\|\partial_{y} \theta(t)\right\|_{L^{2}}^{2}\right)+\kappa_{1}\left\|\partial_{x x} \theta\right\|_{L^{2}}^{2}+\kappa_{1}\left\|\partial_{x y} \theta\right\|_{L^{2}}^{2} \\
& \quad \leq C\left(\left\|\partial_{y} u_{1}\right\|_{L^{2}}^{2}+\|\omega\|_{L^{2}}^{2}+\left\|u_{1}\right\|_{L^{2}}^{2}\left\|\partial_{y} u_{1}\right\|_{L^{2}}^{2}+1\right)\left(\left\|\partial_{x} \theta\right\|_{L^{2}}^{2}+\left\|\partial_{y} \theta\right\|_{L^{2}}^{2}\right)+C\|\omega\|_{L^{2}}^{2}
\end{aligned}
$$

Then (2.18), together with Gronwall's inequality, immediately yields

$$
\|\nabla \theta\|_{L^{\infty}\left(\left[0, T ; L^{2}\left(\mathbb{R}^{2}\right)\right)\right.}+\kappa_{1}\left\|\nabla \partial_{x} \theta\right\|_{L^{2}\left([0, T) ; L^{2}\left(\mathbb{R}^{2}\right)\right)} \leq C .
$$

Hence, we finish the proof of Lemma 2.5.

We next prove Theorem 2.3 by using the method of vanishing viscosity. To this end, we consider the following regularized problem:

$$
\left\{\begin{array}{l}
\partial_{t} u_{1}^{\varepsilon}+\left(u^{\varepsilon} \cdot \nabla\right) u_{1}^{\varepsilon}+\partial_{x} \pi^{\varepsilon}=\varepsilon \partial_{x x} u_{1}^{\varepsilon}+\mu_{2} \partial_{y y} u_{1}^{\varepsilon}, \\
\partial_{t} u_{2}^{\varepsilon}+\left(u^{\varepsilon} \cdot \nabla\right) u_{2}^{\varepsilon}+\partial_{y} \pi^{\varepsilon}=\varepsilon \partial_{x x} u_{2}^{\varepsilon}+\varepsilon \partial_{y y} u_{2}^{\varepsilon}+\theta^{\varepsilon} \\
\partial_{t} \theta^{\varepsilon}+\left(u^{\varepsilon} \cdot \nabla\right) \theta^{\varepsilon}=\kappa_{1} \partial_{x x} \theta^{\varepsilon}+\varepsilon \partial_{y y} \theta^{\varepsilon}+u_{2}^{\varepsilon} \\
\nabla \cdot u^{\varepsilon}=0
\end{array}\right.
$$

with the smooth initial data

$$
u_{1}^{\varepsilon}(x, y, 0)=\varphi_{\varepsilon} * u_{1}^{0}, \quad u_{2}^{\varepsilon}(x, y, 0)=\varphi_{\varepsilon} * u_{2}^{0}, \quad \theta^{\varepsilon}(x, y, 0)=\varphi_{\varepsilon} * \theta^{0},
$$

where $u^{\varepsilon}=\left(u_{1}^{\varepsilon}, u_{2}^{\varepsilon}\right)$, “*” is the usual convolution operator, and $\varphi_{\varepsilon}(x, y)=\varepsilon^{-2} \varphi(x / \varepsilon, y / \varepsilon)$ is the standard mollifier with width $\varepsilon$, which satisfying

$$
\varphi \geq 0, \varphi \in C_{0}^{\infty}\left(\mathbb{R}^{2}\right), \quad \iint_{\mathbb{R}^{2}} \varphi d x d y=1
$$

Now, an application of the result of Sect. 3 in [32] in p. 934 shows that, for any $T>0$, there exists a unique global smooth solution $\left(u_{1}^{\varepsilon}, u_{2}^{\varepsilon}, \theta^{\varepsilon}\right)$ of $(2.20),(2.21)$ on $\mathbb{R}^{2} \times(0, T)$ satisfying the global bounds stated in Lemmas $2.4-2.5$ which are uniform in $\varepsilon$. So, by standard 
compactness arguments, we can extract a subsequence $\left(u_{1}^{\varepsilon_{j}}, u_{2}^{\varepsilon_{j}}, \theta^{\varepsilon_{j}}\right)$ and pass to the limit as $j \rightarrow \infty$ to find that the limit function $\left(u_{1}, u_{2}, \theta\right)$ is indeed a global weak solution of the problem (1.2)-(1.3) with $\mu_{1}=0, \mu_{2}>0, \mu_{3}=0, \mu_{4}=0, \kappa_{1}>0, \kappa_{2}=0$. The uniqueness of the solution $\left(u_{1}, u_{2}, \theta\right)$ satisfying the condition stated in Theorem 2.3 can be proved in a very standard way, and for simplicity we omit the details here. The proof of Theorem 2.3 is therefore completed.

\section{Global regularity criteria for weak solution of the Bénard system with vertical dissipation and horizontal thermal diffusivity}

The issue of whether the 2D Bénard system always possesses global (in time) classical solutions can be difficult when there is only partial dissipation. Therefore, the goal of this section is to establish two global regularity criteria for the weak solution of the 2D Bénard system with vertical dissipation in the first component of velocity field and horizontal thermal diffusivity.

Theorem 3.1 Let $\mu_{1}=0, \mu_{2}>0, \mu_{3}=0, \mu_{4}=0, \kappa_{1}>0, \kappa_{2}=0$. Suppose that $u_{1}^{0}, u_{2}^{0}, \theta^{0} \in$ $H^{2}\left(\mathbb{R}^{2}\right)$ and $\partial_{x} u_{1}^{0}+\partial_{y} u_{2}^{0}=0$. If the condition holds that

$$
\int_{0}^{T}\left\|\partial_{x x} u_{2}(\tau)\right\|_{L^{4}\left(\mathbb{R}^{2}\right)} d \tau<\infty
$$

for any fixed $T>0$, then the problem (1.2)-(1.3) admits a global classical solution $\left(u_{1}, u_{2}, \theta\right)$, which obeys

$$
\begin{aligned}
u_{1}, u_{2}, \theta & \in L^{\infty}\left([0, T) ; H^{2}\left(\mathbb{R}^{2}\right)\right), \\
\partial_{x y y} u_{1} & \in L^{2}\left([0, T) ; L^{2}\left(\mathbb{R}^{2}\right)\right), \quad \partial_{y} u_{1}, \partial_{x} \theta \in L^{2}\left([0, T) ; H^{2}\left(\mathbb{R}^{2}\right)\right) .
\end{aligned}
$$

Proof Applying $\nabla$ to Eq. (2.6) ${ }_{1}$ and taking the $L^{2}$-inner product with $\nabla \omega$, and integrating by parts, we obtain

$$
\begin{gathered}
\frac{1}{2} \frac{d}{d t}\|\nabla \omega(t)\|_{L^{2}}^{2}+\mu_{2}\left\|\partial_{x x y} u_{1}\right\|_{L^{2}}^{2}+2 \mu_{2}\left\|\partial_{x y y} u_{1}\right\|_{L^{2}}^{2}+\mu_{2}\left\|\partial_{y y y} u_{1}\right\|_{L^{2}}^{2} \\
\quad=-\iint_{\mathbb{R}^{2}} \nabla[(u \cdot \nabla) \omega] \cdot \nabla \omega d x d y+\iint_{\mathbb{R}^{2}} \nabla \partial_{x} \theta \nabla \omega d x d y .
\end{gathered}
$$

By the divergence-free condition, we further split the first term of (3.2) into four terms;

$$
\begin{aligned}
- & \iint_{\mathbb{R}^{2}} \nabla[(u \cdot \nabla) \omega] \cdot \nabla \omega d x d y \\
= & -\iint_{\mathbb{R}^{2}} \partial_{x} u_{1}\left(\partial_{x} \omega\right)^{2} d x d y-\iint_{\mathbb{R}^{2}} \partial_{x} u_{2} \partial_{x} \omega \partial_{y} \omega d x d y \\
& \quad-\iint_{\mathbb{R}^{2}} \partial_{y} u_{1} \partial_{x} \omega \partial_{y} \omega d x d y-\iint_{\mathbb{R}^{2}} \partial_{y} u_{2}\left(\partial_{y} \omega\right)^{2} d x d y .
\end{aligned}
$$

Differentiating Eqs. (2.6) $)_{2}$ and (2.6) $)_{3}$ with respect to $x$ and $y$ and multiplying the resulting equations by $\partial_{x x} \theta$ and $\partial_{y y} \theta$, respectively, we deduce after integrating by parts over $\mathbb{R}^{2}$ 
that

$$
\begin{gathered}
\frac{1}{2} \frac{d}{d t}\left(\left\|\partial_{x x} \theta(t)\right\|_{L^{2}}^{2}+\left\|\partial_{y y} \theta(t)\right\|_{L^{2}}^{2}\right)+\kappa_{1}\left\|\partial_{x x x} \theta\right\|_{L^{2}}^{2}+\kappa_{1}\left\|\partial_{x y y} \theta\right\|_{L^{2}}^{2} \\
=-\iint_{\mathbb{R}^{2}} \partial_{x x}[(u \cdot \nabla) \theta] \partial_{x x} \theta d x d y+\iint_{\mathbb{R}^{2}} \partial_{x x} u_{2} \partial_{x x} \theta d x d y \\
-\iint_{\mathbb{R}^{2}} \partial_{y y}[(u \cdot \nabla) \theta] \partial_{y y} \theta d x d y+\iint_{\mathbb{R}^{2}} \partial_{y y} u_{2} \partial_{y y} \theta d x d y .
\end{gathered}
$$

We now turn to the first and third terms of (3.4). Again we write them out explicitly as

$$
\begin{aligned}
& -\iint_{\mathbb{R}^{2}} \partial_{x x}[(u \cdot \nabla) \theta] \partial_{x x} \theta d x d y \\
& =\iint_{\mathbb{R}^{2}} \partial_{x}[(u \cdot \nabla) \theta] \partial_{x x x} \theta d x d y \\
& =-\iint_{\mathbb{R}^{2}} \partial_{x} u_{1} \partial_{x} \theta \partial_{x x x} \theta d x d y+\iint_{\mathbb{R}^{2}} u_{1} \partial_{x x} \theta \partial_{x x x} \theta d x d y \\
& \quad+\iint_{\mathbb{R}^{2}} \partial_{x} u_{2} \partial_{y} \theta \partial_{x x x} \theta d x d y+\iint_{\mathbb{R}^{2}} u_{2} \partial_{x y} \theta \partial_{x x x} \theta d x d y, \\
& -\iint_{\mathbb{R}^{2}} \partial_{y y}[(u \cdot \nabla) \theta] \partial_{y y} \theta d x d y \\
& =\iint_{\mathbb{R}^{2}} \partial_{y}[(u \cdot \nabla) \theta] \partial_{y y y} \theta d x d y \\
& =\iint_{\mathbb{R}^{2}} \partial_{y} u_{1} \partial_{x} \theta \partial_{y y y} \theta d x d y+\iint_{\mathbb{R}^{2}} u_{1} \partial_{x y} \theta \partial_{y y y} \theta d x d y \\
& \quad+\iint_{\mathbb{R}^{2}} \partial_{y} u_{2} \partial_{y} \theta \partial_{y y y} \theta d x d y+\iint_{\mathbb{R}^{2}} u_{2} \partial_{y y} \theta \partial_{y y y} \theta d x d y .
\end{aligned}
$$

Combining with (3.2)-(3.6) leads to

$$
\begin{aligned}
\frac{1}{2} \frac{d}{d t}( & \left.\|\nabla \omega(t)\|_{L^{2}}^{2}+\left\|\partial_{x x} \theta(t)\right\|_{L^{2}}^{2}+\left\|\partial_{y y} \theta(t)\right\|_{L^{2}}^{2}\right)+\mu_{2}\left\|\partial_{x x y} u_{1}\right\|_{L^{2}}^{2}+2 \mu_{2}\left\|\partial_{x y y} u_{1}\right\|_{L^{2}}^{2} \\
& +\mu_{2}\left\|\partial_{y y y} u_{1}\right\|_{L^{2}}^{2}+\kappa_{1}\left\|\partial_{x x x} \theta\right\|_{L^{2}}^{2}+\kappa_{1}\left\|\partial_{x y y} \theta\right\|_{L^{2}}^{2} \\
= & -\iint_{\mathbb{R}^{2}} \partial_{x} u_{1}\left(\partial_{x} \omega\right)^{2} d x d y-\iint_{\mathbb{R}^{2}} \partial_{x} u_{2} \partial_{x} \omega \partial_{y} \omega d x d y-\iint_{\mathbb{R}^{2}} \partial_{y} u_{1} \partial_{x} \omega \partial_{y} \omega d x d y \\
& -\iint_{\mathbb{R}^{2}} \partial_{y} u_{2}\left(\partial_{y} \omega\right)^{2} d x d y+\iint_{\mathbb{R}^{2}} \nabla \partial_{x} \theta \nabla \omega d x d y+\iint_{\mathbb{R}^{2}} \partial_{x} u_{1} \partial_{x} \theta \partial_{x x x} \theta d x d y \\
& +\iint_{\mathbb{R}^{2}} \partial_{x} u_{2} \partial_{y} \theta \partial_{x x x} \theta d x d y+\iint_{\mathbb{R}^{2}} u_{2} \partial_{x y} \theta \partial_{x x x} \theta d x d y+\iint_{\mathbb{R}^{2}} \partial_{y} u_{1} \partial_{x} \theta \partial_{y y y} \theta d x d y \\
& +\iint_{\mathbb{R}^{2}} u_{1} \partial_{x y} \theta \partial_{y y y} \theta d x d y+\iint_{\mathbb{R}^{2}} \partial_{y} u_{2} \partial_{y} \theta \partial_{y y y} \theta d x d y+\iint_{\mathbb{R}^{2}} \partial_{x x} u_{2} \partial_{x x} \theta d x d y \\
& +\iint_{\mathbb{R}^{2}} \partial_{y y} u_{2} \partial_{y y} \theta d x d y+\iint_{\mathbb{R}^{2}} u_{1} \partial_{x x} \theta \partial_{x x x} \theta d x d y+\iint_{\mathbb{R}^{2}} u_{2} \partial_{y y} \theta \partial_{y y y} \theta d x d y \\
= & =\sum_{l=1}^{15} J_{l}
\end{aligned}
$$


We now estimate $J_{1}$ through $J_{7}$. Applying Lemma 2.1, the Cauchy-Schwarz inequality and Young's inequality, we have

$$
\begin{aligned}
J_{1}= & -\iint_{\mathbb{R}^{2}} \partial_{x} u_{1}\left(\partial_{x} \omega\right)^{2} d x d y \\
= & -\iint_{\mathbb{R}^{2}} \partial_{x} u_{1}\left(\partial_{x x} u_{2}\right)^{2} d x d y-\iint_{\mathbb{R}^{2}} \partial_{x} u_{1}\left(\partial_{x y} u_{1}\right)^{2} d x d y \\
& +2 \iint_{\mathbb{R}^{2}} \partial_{x} u_{1} \partial_{x x} u_{2} \partial_{x y} u_{1} d x d y \\
\leq & C\left\|\partial_{x} u_{1}\right\|_{L^{4}}\left\|\partial_{x x} u_{2}\right\|_{L^{2}}\left\|\partial_{x x} u_{2}\right\|_{L^{4}} \\
& +C\left\|\partial_{x} u_{1}\right\|_{L^{2}}\left\|\partial_{x y} u_{1}\right\|_{L^{2}}^{\frac{1}{2}}\left\|\partial_{x x y} u_{1}\right\|_{L^{2}}^{\frac{1}{2}}\left\|\partial_{x y} u_{1}\right\|_{L^{2}}^{\frac{1}{2}}\left\|\partial_{x y y} u_{1}\right\|_{L^{2}}^{\frac{1}{2}} \\
& +C\left\|\partial_{x} u_{1}\right\|_{L^{4}}\left\|\partial_{x x} u_{2}\right\|_{L^{4}}\left\|\partial_{x y} u_{1}\right\|_{L^{2}} \\
\leq & C\left\|\partial_{x} u_{1}\right\|_{L^{2}}^{\frac{1}{2}}\left\|\nabla \partial_{x} u_{1}\right\|_{L^{2}}^{\frac{1}{2}}\left\|\partial_{x x} u_{2}\right\|_{L^{2}}\left\|\partial_{x x} u_{2}\right\|_{L^{4}} \\
& +C\|\omega\|_{L^{2}}\left\|\partial_{x y} u_{1}\right\|_{L^{2}}^{\frac{1}{2}}\left\|\partial_{x x y} u_{1}\right\|_{L^{2}}^{\frac{1}{2}}\left\|\partial_{x y} u_{1}\right\|_{L^{2}}^{\frac{1}{2}}\left\|\partial_{x y y} u_{1}\right\|_{L^{2}}^{\frac{1}{2}} \\
& +C\left\|\partial_{x} u_{1}\right\|_{L^{2}}^{\frac{1}{2}}\left\|\nabla \partial_{x} u_{1}\right\|_{L^{2}}^{\frac{1}{2}}\left\|\partial_{x x} u_{2}\right\|_{L^{4}}\left\|\partial_{x y} u_{1}\right\|_{L^{2}} \\
\leq & C\|\omega\|_{L^{2}}^{\frac{1}{2}}\|\nabla \omega\|_{L^{2}}^{\frac{3}{2}}\left\|\partial_{x x} u_{2}\right\|_{L^{4}}+C\|\omega\|_{L^{2}}\|\nabla \omega\|_{L^{2}}\left\|\partial_{x x y} u_{1}\right\|_{L^{2}}^{\frac{1}{2}}\left\|\partial_{x y y} u_{1}\right\|_{L^{2}}^{\frac{1}{2}} \\
& +C\|\omega\|_{L^{2}}^{\frac{1}{2}}\|\nabla \omega\|_{L^{2}}^{\frac{3}{2}}\left\|\partial_{x x} u_{2}\right\|_{L^{4}} \\
\leq & \frac{\mu_{2}}{16}\left\|\partial_{x x y} u_{1}\right\|_{L^{2}}^{2}+\frac{\mu_{2}}{2}\left\|\partial_{x y y} u_{1}\right\|_{L^{2}}^{2}+C\left(\left\|\partial_{x x} u_{2}\right\|_{L^{4}}+\|\omega\|_{L^{2}}^{2}\right)\|\nabla \omega\|_{L^{2}}^{2} \\
& +C\left\|\partial_{x x} u_{2}\right\|_{L^{4}}\|\omega\|_{L^{2}}^{2},
\end{aligned}
$$

where we have utilized the inequality

$$
\|f\|_{L^{4}} \leq\|f\|_{L^{2}}^{\frac{1}{2}}\|\nabla f\|_{L^{2}}^{\frac{1}{2}}
$$

Similarly, invoking Lemma 2.1 and (3.9), the Cauchy-Schwarz inequality and Young's inequality, the remainder terms can be estimated as follows:

$$
\begin{aligned}
J_{2}= & -\iint_{\mathbb{R}^{2}} \partial_{x} u_{2} \partial_{x} \omega \partial_{y} \omega d x d y \\
= & -\iint_{\mathbb{R}^{2}} \partial_{x} u_{2} \partial_{x x} u_{2} \partial_{x y} u_{2} d x d y+\iint_{\mathbb{R}^{2}} \partial_{x} u_{2} \partial_{x y} u_{1} \partial_{x y} u_{2} d x d y \\
& +\iint_{\mathbb{R}^{2}} \partial_{x} u_{2} \partial_{x x} u_{2} \partial_{y y} u_{1} d x d y-\iint_{\mathbb{R}^{2}} \partial_{x} u_{2} \partial_{x y} u_{1} \partial_{y y} u_{1} d x d y \\
\leq & C\left\|\partial_{x} u_{2}\right\|_{L^{4}}\left\|\partial_{x x} u_{2}\right\|_{L^{4}}\left\|\partial_{x y} u_{2}\right\|_{L^{2}} \\
& +C\left\|\partial_{x} u_{2}\right\|_{L^{2}}\left\|\partial_{x y} u_{1}\right\|_{L^{2}}^{\frac{1}{2}}\left\|\partial_{x x y} u_{1}\right\|_{L^{2}}^{\frac{1}{2}}\left\|\partial_{x y} u_{2}\right\|_{L^{2}}^{\frac{1}{2}}\left\|\partial_{x y y} u_{2}\right\|_{L^{2}}^{\frac{1}{2}} \\
& +C\left\|\partial_{x} u_{2}\right\|_{L^{4}}\left\|\partial_{x x} u_{2}\right\|_{L^{4}}\left\|\partial_{y y} u_{1}\right\|_{L^{2}} \\
& +C\left\|\partial_{x} u_{2}\right\|_{L^{2}}\left\|\partial_{x y} u_{1}\right\|_{L^{2}}^{\frac{1}{2}}\left\|\partial_{x x y} u_{1}\right\|_{L^{2}}^{\frac{1}{2}}\left\|\partial_{y y} u_{1}\right\|_{L^{2}}^{\frac{1}{2}}\left\|\partial_{y y y} u_{1}\right\|_{L^{2}}^{\frac{1}{2}} \\
\leq & C\left\|\partial_{x} u_{2}\right\|_{L^{2}}^{\frac{1}{2}}\left\|\nabla \partial_{x} u_{2}\right\|_{L^{2}}^{\frac{1}{2}}\left\|\partial_{x x} u_{2}\right\|_{L^{4}}\|\nabla \omega\|_{L^{2}}
\end{aligned}
$$




$$
\begin{aligned}
& +C\|\omega\|_{L^{2}}\|\nabla \omega\|_{L^{2}}^{\frac{1}{2}}\left\|\partial_{x x y} u_{1}\right\|_{L^{2}}^{\frac{1}{2}}\|\nabla \omega\|_{L^{2}}^{\frac{1}{2}}\left\|\partial_{x x y} u_{1}\right\|_{L^{2}}^{\frac{1}{2}} \\
& +C\left\|\partial_{x} u_{2}\right\|_{L^{2}}^{\frac{1}{2}}\left\|\nabla \partial_{x} u_{2}\right\|_{L^{2}}^{\frac{1}{2}}\left\|\partial_{x x} u_{2}\right\|_{L^{4}}\|\nabla \omega\|_{L^{2}} \\
& +C\|\omega\|_{L^{2}}\|\nabla \omega\|_{L^{2}}^{\frac{1}{2}}\left\|\partial_{x x y} u_{1}\right\|_{L^{2}}^{\frac{1}{2}}\|\nabla \omega\|_{L^{2}}^{\frac{1}{2}}\left\|\partial_{y y y} u_{1}\right\|_{L^{2}}^{\frac{1}{2}} \\
& \leq C\|\omega\|_{L^{2}}^{\frac{1}{2}}\|\nabla \omega\|_{L^{2}}^{\frac{3}{2}}\left\|\partial_{x x} u_{2}\right\|_{L^{4}}+C\|\omega\|_{L^{2}}\|\nabla \omega\|_{L^{2}}\left\|\partial_{x x y} u_{1}\right\|_{L^{2}} \\
& +C\|\omega\|_{L^{2}}^{\frac{1}{2}}\|\nabla \omega\|_{L^{2}}^{\frac{3}{2}}\left\|\partial_{x x} u_{2}\right\|_{L^{4}}+C\|\omega\|_{L^{2}}\|\nabla \omega\|_{L^{2}}\left\|\partial_{x x y} u_{1}\right\|_{L^{2}}^{\frac{1}{2}}\left\|\partial_{y y y} u_{1}\right\|_{L^{2}}^{\frac{1}{2}} \\
& \leq \frac{\mu_{2}}{8}\left\|\partial_{x x y} u_{1}\right\|_{L^{2}}^{2}+\frac{\mu_{2}}{12}\left\|\partial_{y y y} u_{1}\right\|_{L^{2}}^{2}+C\left(\left\|\partial_{x x} u_{2}\right\|_{L^{4}}+\|\omega\|_{L^{2}}^{2}\right)\|\nabla \omega\|_{L^{2}}^{2} \\
& +C\left\|\partial_{x x} u_{2}\right\|_{L^{4}}\|\omega\|_{L^{2}}^{2} \text {, } \\
& J_{3}=-\iint_{\mathbb{R}^{2}} \partial_{y} u_{1} \partial_{x} \omega \partial_{y} \omega d x d y \\
& =-\iint_{\mathbb{R}^{2}} \partial_{y} u_{1} \partial_{x x} u_{2} \partial_{x y} u_{2} d x d y+\iint_{\mathbb{R}^{2}} \partial_{y} u_{1} \partial_{x x} u_{2} \partial_{y y} u_{1} d x d y \\
& +\iint_{\mathbb{R}^{2}} \partial_{y} u_{1} \partial_{x y} u_{1} \partial_{x y} u_{2} d x d y-\iint_{\mathbb{R}^{2}} \partial_{y} u_{1} \partial_{x y} u_{1} \partial_{y y} u_{1} d x d y \\
& \leq C\left\|\partial_{y} u_{1}\right\|_{L^{4}}\left\|\partial_{x x} u_{2}\right\|_{L^{4}}\left\|\partial_{x y} u_{2}\right\|_{L^{2}}+C\left\|\partial_{y} u_{1}\right\|_{L^{4}}\left\|\partial_{x x} u_{2}\right\|_{L^{4}}\left\|\partial_{y y} u_{1}\right\|_{L^{2}} \\
& +C\left\|\partial_{y} u_{1}\right\|_{L^{2}}\left\|\partial_{x y} u_{1}\right\|_{L^{2}}^{\frac{1}{2}}\left\|\partial_{x x y} u_{1}\right\|_{L^{2}}^{\frac{1}{2}}\left\|\partial_{x y} u_{2}\right\|_{L^{2}}^{\frac{1}{2}}\left\|\partial_{x y y} u_{2}\right\|_{L^{2}}^{\frac{1}{2}} \\
& +C\left\|\partial_{y} u_{1}\right\|_{L^{2}}\left\|\partial_{x y} u_{1}\right\|_{L^{2}}^{\frac{1}{2}}\left\|\partial_{x x y} u_{1}\right\|_{L^{2}}^{\frac{1}{2}}\left\|\partial_{y y} u_{1}\right\|_{L^{2}}^{\frac{1}{2}}\left\|\partial_{y y y} u_{1}\right\|_{L^{2}}^{\frac{1}{2}} \\
& \leq C\left\|\partial_{y} u_{1}\right\|_{L^{2}}^{\frac{1}{2}}\left\|\nabla \partial_{y} u_{1}\right\|_{L^{2}}^{\frac{1}{2}}\left\|\partial_{x x} u_{2}\right\|_{L^{4}}\|\nabla \omega\|_{L^{2}} \\
& +C\left\|\partial_{y} u_{1}\right\|_{L^{2}}^{\frac{1}{2}}\left\|\nabla \partial_{y} u_{1}\right\|_{L^{2}}^{\frac{1}{2}}\left\|\partial_{x x} u_{2}\right\|_{L^{4}}\|\nabla \omega\|_{L^{2}} \\
& +C\left\|\partial_{y} u_{1}\right\|_{L^{2}}\|\nabla \omega\|_{L^{2}}^{\frac{1}{2}}\left\|\partial_{x x y} u_{1}\right\|_{L^{2}}^{\frac{1}{2}}\|\nabla \omega\|_{L^{2}}^{\frac{1}{2}}\left\|\partial_{x x y} u_{1}\right\|_{L^{2}}^{\frac{1}{2}} \\
& +C\left\|\partial_{y} u_{1}\right\|_{L^{2}}\|\nabla \omega\|_{L^{2}}^{\frac{1}{2}}\left\|\partial_{x x y} u_{1}\right\|_{L^{2}}^{\frac{1}{2}}\|\nabla \omega\|_{L^{2}}^{\frac{1}{2}}\left\|\partial_{y y y} u_{1}\right\|_{L^{2}}^{\frac{1}{2}} \\
& \leq C\left\|\partial_{y} u_{1}\right\|_{L^{2}}^{\frac{1}{2}}\|\nabla \omega\|_{L^{2}}^{\frac{3}{2}}\left\|\partial_{x x} u_{2}\right\|_{L^{4}}+C\left\|\partial_{y} u_{1}\right\|_{L^{2}}^{\frac{1}{2}}\|\nabla \omega\|_{L^{2}}^{\frac{3}{2}}\left\|\partial_{x x} u_{2}\right\|_{L^{4}} \\
& +C\left\|\partial_{y} u_{1}\right\|_{L^{2}}\|\nabla \omega\|_{L^{2}}\left\|\partial_{x x y} u_{1}\right\|_{L^{2}}+C\left\|\partial_{y} u_{1}\right\|_{L^{2}}\|\nabla \omega\|_{L^{2}}\left\|\partial_{x x y} u_{1}\right\|_{L^{2}}^{\frac{1}{2}}\left\|\partial_{y y y} u_{1}\right\|_{L^{2}}^{\frac{1}{2}} \\
& \leq \frac{\mu_{2}}{8}\left\|\partial_{x x y} u_{1}\right\|_{L^{2}}^{2}+\frac{\mu_{2}}{12}\left\|\partial_{y y y} u_{1}\right\|_{L^{2}}^{2}+C\left(\left\|\partial_{x x} u_{2}\right\|_{L^{4}}+\left\|\partial_{y} u_{1}\right\|_{L^{2}}^{2}\right)\|\nabla \omega\|_{L^{2}}^{2} \\
& +C\left\|\partial_{x x} u_{2}\right\|_{L^{4}}\left\|\partial_{y} u_{1}\right\|_{L^{2}}^{2}, \\
& J_{4}=-\iint_{\mathbb{R}^{2}} \partial_{y} u_{2}\left(\partial_{y} \omega\right)^{2} d x d y \\
& =2 \iint_{\mathbb{R}^{2}} u_{2} \partial_{x y} u_{2} \partial_{x y y} u_{2} d x d y+2 \iint_{\mathbb{R}^{2}} u_{2} \partial_{y y} u_{1} \partial_{y y y} u_{1} d x d y \\
& +2 \iint_{\mathbb{R}^{2}} u_{2} \partial_{x y y} u_{2} \partial_{y y} u_{1} d x d y+2 \iint_{\mathbb{R}^{2}} u_{2} \partial_{x y} u_{2} \partial_{y y y} u_{1} d x d y \\
& \leq C\left\|\partial_{x y y} u_{2}\right\|_{L^{2}}\left\|u_{2}\right\|_{L^{2}}^{\frac{1}{2}}\left\|\partial_{x} u_{2}\right\|_{L^{2}}^{\frac{1}{2}}\left\|\partial_{x y} u_{2}\right\|_{L^{2}}^{\frac{1}{2}}\left\|\partial_{x y y} u_{2}\right\|_{L^{2}}^{\frac{1}{2}} \\
& +C\left\|\partial_{y y y} u_{1}\right\|_{L^{2}}\left\|u_{2}\right\|_{L^{2}}^{\frac{1}{2}}\left\|\partial_{x} u_{2}\right\|_{L^{2}}^{\frac{1}{2}}\left\|\partial_{y y} u_{1}\right\|_{L^{2}}^{\frac{1}{2}}\left\|\partial_{y y y} u_{1}\right\|_{L^{2}}^{\frac{1}{2}}
\end{aligned}
$$




$$
\begin{aligned}
& +C\left\|\partial_{x y y} u_{2}\right\|_{L^{2}}\left\|u_{2}\right\|_{L^{2}}^{\frac{1}{2}}\left\|\partial_{x} u_{2}\right\|_{L^{2}}^{\frac{1}{2}}\left\|\partial_{y y} u_{1}\right\|_{L^{2}}^{\frac{1}{2}}\left\|\partial_{y y y} u_{1}\right\|_{L^{2}}^{\frac{1}{2}} \\
& +C\left\|\partial_{y y y} u_{1}\right\|_{L^{2}}\left\|u_{2}\right\|_{L^{2}}^{\frac{1}{2}}\left\|\partial_{x} u_{2}\right\|_{L^{2}}^{\frac{1}{2}}\left\|\partial_{x y} u_{2}\right\|_{L^{2}}^{\frac{1}{2}}\left\|\partial_{x y y} u_{2}\right\|_{L^{2}}^{\frac{1}{2}} \\
& \leq C\left\|\partial_{x x y} u_{1}\right\|_{L^{2}}\left\|u_{2}\right\|_{L^{2}}^{\frac{1}{2}}\|\omega\|_{L^{2}}^{\frac{1}{2}}\|\nabla \omega\|_{L^{2}}^{\frac{1}{2}}\left\|\partial_{x x y} u_{1}\right\|_{L^{2}}^{\frac{1}{2}} \\
& +C\left\|\partial_{y y y} u_{1}\right\|_{L^{2}}\left\|u_{2}\right\|_{L^{2}}^{\frac{1}{2}}\|\omega\|_{L^{2}}^{\frac{1}{2}}\|\nabla \omega\|_{L^{2}}^{\frac{1}{2}}\left\|\partial_{y y y} u_{1}\right\|_{L^{2}}^{\frac{1}{2}} \\
& +C\left\|\partial_{x x y} u_{1}\right\|_{L^{2}}\left\|u_{2}\right\|_{L^{2}}^{\frac{1}{2}}\|\omega\|_{L^{2}}^{\frac{1}{2}}\|\nabla \omega\|_{L^{2}}^{\frac{1}{2}}\left\|\partial_{y y y} u_{1}\right\|_{L^{2}}^{\frac{1}{2}} \\
& +C\left\|\partial_{y y y} u_{1}\right\|_{L^{2}}\left\|u_{2}\right\|_{L^{2}}^{\frac{1}{2}}\|\omega\|_{L^{2}}^{\frac{1}{2}}\|\nabla \omega\|_{L^{2}}^{\frac{1}{2}}\left\|\partial_{x x y} u_{1}\right\|_{L^{2}}^{\frac{1}{2}} \\
& \leq \frac{3 \mu_{2}}{16}\left\|\partial_{x x y} u_{1}\right\|_{L^{2}}^{2}+\frac{3 \mu_{2}}{12}\left\|\partial_{y y y} u_{1}\right\|_{L^{2}}^{2}+C\left\|u_{2}\right\|_{L^{2}}^{2}\|\omega\|_{L^{2}}^{2}\|\nabla \omega\|_{L^{2}}^{2} \text {, } \\
& J_{5}=\iint_{\mathbb{R}^{2}} \nabla \partial_{x} \theta \nabla \omega d x d y \leq C\left(\left\|\partial_{x x} \theta\right\|_{L^{2}}^{2}+\left\|\partial_{y y} \theta\right\|_{L^{2}}^{2}+\|\nabla \omega\|_{L^{2}}^{2}\right) \text {, } \\
& J_{6}=\iint_{\mathbb{R}^{2}} \partial_{x} u_{1} \partial_{x} \theta \partial_{x x x} \theta d x d y \\
& \leq C\left\|\partial_{x x x} \theta\right\|_{L^{2}}\left\|\partial_{x} u_{1}\right\|_{L^{2}}^{\frac{1}{2}}\left\|\partial_{x y} u_{1}\right\|_{L^{2}}^{\frac{1}{2}}\left\|\partial_{x} \theta\right\|_{L^{2}}^{\frac{1}{2}}\left\|\partial_{x x} \theta\right\|_{L^{2}}^{\frac{1}{2}} \\
& \leq \frac{\kappa_{1}}{6}\left\|\partial_{x x x} \theta\right\|_{L^{2}}^{2}+C\|\omega\|_{L^{2}}^{2}\|\nabla \omega\|_{L^{2}}^{2}+C\left\|\partial_{x} \theta\right\|_{L^{2}}^{2}\left\|\partial_{x x} \theta\right\|_{L^{2}}^{2}, \\
& J_{7}=\iint_{\mathbb{R}^{2}} \partial_{x} u_{2} \partial_{y} \theta \partial_{x x x} \theta d x d y \\
& \leq C\left\|\partial_{x x x} \theta\right\|_{L^{2}}\left\|\partial_{x} u_{2}\right\|_{L^{2}}^{\frac{1}{2}}\left\|\partial_{x x} u_{2}\right\|_{L^{2}}^{\frac{1}{2}}\left\|\partial_{y} \theta\right\|_{L^{2}}^{\frac{1}{2}}\left\|\partial_{y y} \theta\right\|_{L^{2}}^{\frac{1}{2}} \\
& \leq \frac{\kappa_{1}}{6}\left\|\partial_{x x x} \theta\right\|_{L^{2}}^{2}+C\|\omega\|_{L^{2}}^{2}\|\nabla \omega\|_{L^{2}}^{2}+C\left\|\partial_{y} \theta\right\|_{L^{2}}^{2}\left\|\partial_{y y} \theta\right\|_{L^{2}}^{2} \text {, } \\
& J_{8}=\iint_{\mathbb{R}^{2}} u_{2} \partial_{x y} \theta \partial_{x x x} \theta d x d y \\
& \leq C\left\|\partial_{x x x} \theta\right\|_{L^{2}}\left\|u_{2}\right\|_{L^{2}}^{\frac{1}{2}}\left\|\partial_{x} u_{2}\right\|_{L^{2}}^{\frac{1}{2}}\left\|\partial_{x y} \theta\right\|_{L^{2}}^{\frac{1}{2}}\left\|\partial_{x y y} \theta\right\|_{L^{2}}^{\frac{1}{2}} \\
& \leq \frac{\kappa_{1}}{6}\left\|\partial_{x x x} \theta\right\|_{L^{2}}^{2}+\frac{\kappa_{1}}{14}\left\|\partial_{x y y} \theta\right\|_{L^{2}}^{2}+C\left\|u_{2}\right\|_{L^{2}}^{2}\|\omega\|_{L^{2}}^{2}\left(\left\|\partial_{x x} \theta\right\|_{L^{2}}^{2}+\left\|\partial_{y y} \theta\right\|_{L^{2}}^{2}\right),
\end{aligned}
$$

where we have used the fact $\left\|\partial_{x y} \theta\right\|_{L^{2}}^{2} \leq C\left(\left\|\partial_{x x} \theta\right\|_{L^{2}}^{2}+\left\|\partial_{y y} \theta\right\|_{L^{2}}^{2}\right)$;

$$
\begin{aligned}
J_{9}= & \iint_{\mathbb{R}^{2}} \partial_{y} u_{1} \partial_{x} \theta \partial_{y y y} \theta d x d y \\
= & -\iint_{\mathbb{R}^{2}} \partial_{y y} u_{1} \partial_{x} \theta \partial_{y y} \theta d x d y-\iint_{\mathbb{R}^{2}} \partial_{y} u_{1} \partial_{x y} \theta \partial_{y y} \theta d x d y \\
\leq & C\left\|\partial_{x} \theta\right\|_{L^{2}}\left\|\partial_{y y} u_{1}\right\|_{L^{2}}^{\frac{1}{2}}\left\|\partial_{y y y} u_{1}\right\|_{L^{2}}^{\frac{1}{2}}\left\|\partial_{y y} \theta\right\|_{L^{2}}^{\frac{1}{2}}\left\|\partial_{x y y} \theta\right\|_{L^{2}}^{\frac{1}{2}} \\
& +C\left\|\partial_{y} u_{1}\right\|_{L^{2}}\left\|\partial_{x y} \theta\right\|_{L^{2}}^{\frac{1}{2}}\left\|\partial_{x y y} \theta\right\|_{L^{2}}^{\frac{1}{2}}\left\|\partial_{y y} \theta\right\|_{L^{2}}^{\frac{1}{2}}\left\|\partial_{x y y} \theta\right\|_{L^{2}}^{\frac{1}{2}} \\
\leq & C\left\|\partial_{x} \theta\right\|_{L^{2}}\|\nabla \omega\|_{L^{2}}^{\frac{1}{2}}\left\|\partial_{y y y} u_{1}\right\|_{L^{2}}^{\frac{1}{2}}\left\|\partial_{y y} \theta\right\|_{L^{2}}^{\frac{1}{2}}\left\|\partial_{x y y} \theta\right\|_{L^{2}}^{\frac{1}{2}} \\
& +C\left\|\partial_{y} u_{1}\right\|_{L^{2}}\left\|\partial_{x y} \theta\right\|_{L^{2}}^{\frac{1}{2}}\left\|\partial_{x y y} \theta\right\|_{L^{2}}\left\|\partial_{y y} \theta\right\|_{L^{2}}^{\frac{1}{2}} \\
\leq & \frac{\mu_{2}}{12}\left\|\partial_{y y y} u_{1}\right\|_{L^{2}}^{2}+\frac{\kappa_{1}}{7}\left\|\partial_{x y y} \theta\right\|_{L^{2}}^{2} \\
& +C\left(\left\|\partial_{x} \theta\right\|_{L^{2}}^{2}+\left\|\partial_{y} u_{1}\right\|_{L^{2}}^{2}\right)\left(\|\nabla \omega\|_{L^{2}}^{2}+\left\|\partial_{x x} \theta\right\|_{L^{2}}^{2}+\left\|\partial_{y y} \theta\right\|_{L^{2}}^{2}\right),
\end{aligned}
$$




$$
\begin{aligned}
& J_{10}=\iint_{\mathbb{R}^{2}} u_{1} \partial_{x y} \theta \partial_{y y y} \theta d x d y \\
& =-\iint_{\mathbb{R}^{2}} \partial_{y} u_{1} \partial_{x y} \theta \partial_{y y} \theta d x d y-\iint_{\mathbb{R}^{2}} u_{1} \partial_{x y y} \theta \partial_{y y} \theta d x d y \\
& \leq C\left\|\partial_{y} u_{1}\right\|_{L^{2}}\left\|\partial_{x y} \theta\right\|_{L^{2}}^{\frac{1}{2}}\left\|\partial_{x y y} \theta\right\|_{L^{2}}^{\frac{1}{2}}\left\|\partial_{y y} \theta\right\|_{L^{2}}^{\frac{1}{2}}\left\|\partial_{x y y} \theta\right\|_{L^{2}}^{\frac{1}{2}} \\
& +C\left\|\partial_{x y y} \theta\right\|_{L^{2}}\left\|u_{1}\right\|_{L^{2}}^{\frac{1}{2}}\left\|\partial_{y} u_{1}\right\|_{L^{2}}^{\frac{1}{2}}\left\|\partial_{y y} \theta\right\|_{L^{2}}^{\frac{1}{2}}\left\|\partial_{x y y} \theta\right\|_{L^{2}}^{\frac{1}{2}} \\
& \leq \frac{\kappa_{1}}{7}\left\|\partial_{x y y} \theta\right\|_{L^{2}}^{2}+C\left(\left\|\partial_{y} u_{1}\right\|_{L^{2}}^{2}+\left\|u_{1}\right\|_{L^{2}}^{2}\left\|\partial_{y} u_{1}\right\|_{L^{2}}^{2}\right)\left(\left\|\partial_{x x} \theta\right\|_{L^{2}}^{2}+\left\|\partial_{y y} \theta\right\|_{L^{2}}^{2}\right), \\
& J_{11}=\iint_{\mathbb{R}^{2}} \partial_{y} u_{2} \partial_{y} \theta \partial_{y y y} \theta d x d y \\
& =-\iint_{\mathbb{R}^{2}} \partial_{y y} u_{2} \partial_{y} \theta \partial_{y y} \theta d x d y-\iint_{\mathbb{R}^{2}} \partial_{y} u_{2} \partial_{y y} \theta \partial_{y y} \theta d x d y \\
& \leq C\left\|\partial_{y} \theta\right\|_{L^{2}}\left\|\partial_{y y} u_{2}\right\|_{L^{2}}^{\frac{1}{2}}\left\|\partial_{y y y} u_{2}\right\|_{L^{2}}^{\frac{1}{2}}\left\|\partial_{y y} \theta\right\|_{L^{2}}^{\frac{1}{2}}\left\|\partial_{x y y} \theta\right\|_{L^{2}}^{\frac{1}{2}} \\
& +C\left\|\partial_{y y} \theta\right\|_{L^{2}}\left\|\partial_{y} u_{2}\right\|_{L^{2}}^{\frac{1}{2}}\left\|\partial_{y y} u_{2}\right\|_{L^{2}}^{\frac{1}{2}}\left\|\partial_{y y} \theta\right\|_{L^{2}}^{\frac{1}{2}}\left\|\partial_{x y y} \theta\right\|_{L^{2}}^{\frac{1}{2}} \\
& \leq C\left\|\partial_{y} \theta\right\|_{L^{2}}\|\nabla \omega\|_{L^{2}}^{\frac{1}{2}}\left\|\partial_{x y y} u_{1}\right\|_{L^{2}}^{\frac{1}{2}}\left\|\partial_{y y} \theta\right\|_{L^{2}}^{\frac{1}{2}}\left\|\partial_{x y y} \theta\right\|_{L^{2}}^{\frac{1}{2}} \\
& +C\left\|\partial_{y y} \theta\right\|_{L^{2}}\|\omega\|_{L^{2}}^{\frac{1}{2}}\left\|\partial_{x y} u_{1}\right\|_{L^{2}}^{\frac{1}{2}}\left\|\partial_{y y} \theta\right\|_{L^{2}}^{\frac{1}{2}}\left\|\partial_{x y y} \theta\right\|_{L^{2}}^{\frac{1}{2}} \\
& \leq \frac{\mu_{2}}{4}\left\|\partial_{x y y} u_{1}\right\|_{L^{2}}^{2}+\frac{\kappa_{1}}{7}\left\|\partial_{x y y} \theta\right\|_{L^{2}}^{2} \\
& +C\left(\left\|\partial_{y} \theta\right\|_{L^{2}}^{2}+\|\omega\|_{L^{2}}^{\frac{2}{3}}\left\|\partial_{x y} u_{1}\right\|_{L^{2}}^{\frac{2}{3}}\right)\left(\|\nabla \omega\|_{L^{2}}^{2}+\left\|\partial_{y y} \theta\right\|_{L^{2}}^{2}\right), \\
& J_{12}=\iint_{\mathbb{R}^{2}} \partial_{x x} u_{2} \partial_{x x} \theta d x d y \leq C\left(\|\nabla \omega\|_{L^{2}}^{2}+\left\|\partial_{x x} \theta\right\|_{L^{2}}^{2}\right) \text {, } \\
& J_{13}=\iint_{\mathbb{R}^{2}} \partial_{y y} u_{2} \partial_{y y} \theta d x d y \leq C\left(\|\nabla \omega\|_{L^{2}}^{2}+\left\|\partial_{y y} \theta\right\|_{L^{2}}^{2}\right),
\end{aligned}
$$

and

$$
\begin{aligned}
J_{14}+J_{15}= & \iint_{\mathbb{R}^{2}} u_{1} \partial_{x x} \theta \partial_{x x x} \theta d x d y+\iint_{\mathbb{R}^{2}} u_{2} \partial_{y y} \theta \partial_{y y y} \theta d x d y \\
= & \iint_{\mathbb{R}^{2}} u_{1} \partial_{x x} \theta \partial_{x x x} \theta d x d y-\iint_{\mathbb{R}^{2}} u_{1} \partial_{y y} \theta \partial_{x y y} \theta d x d y \\
\leq & C\left\|\partial_{x x x} \theta\right\|_{L^{2}}\left\|u_{1}\right\|_{L^{2}}^{\frac{1}{2}}\left\|\partial_{y} u_{1}\right\|_{L^{2}}^{\frac{1}{2}}\left\|\partial_{x x} \theta\right\|_{L^{2}}^{\frac{1}{2}}\left\|\partial_{x x x} \theta\right\|_{L^{2}}^{\frac{1}{2}} \\
& +C\left\|\partial_{x y y} \theta\right\|_{L^{2}}\left\|u_{1}\right\|_{L^{2}}^{\frac{1}{2}}\left\|\partial_{y} u_{1}\right\|_{L^{2}}^{\frac{1}{2}}\left\|\partial_{y y} \theta\right\|_{L^{2}}^{\frac{1}{2}}\left\|\partial_{x y y} \theta\right\|_{L^{2}}^{\frac{1}{2}} \\
\leq & \frac{\kappa_{1}}{8}\left\|\partial_{x x x} \theta\right\|_{L^{2}}^{2}+\frac{\kappa_{1}}{16}\left\|\partial_{x y y} \theta\right\|_{L^{2}}^{2} \\
& +C\left\|u_{1}\right\|_{L^{2}}^{2}\left\|\partial_{y} u_{1}\right\|_{L^{2}}^{2}\left(\left\|\partial_{x x} \theta\right\|_{L^{2}}^{2}+\left\|\partial_{y y} \theta\right\|_{L^{2}}^{2}\right) .
\end{aligned}
$$

Hence, inserting the estimates $J_{1}-J_{15}$ into (3.7), we finally obtain

$$
\begin{aligned}
& \frac{1}{2} \frac{d}{d t}\left(\|\nabla \omega(t)\|_{L^{2}}^{2}+\left\|\partial_{x x} \theta(t)\right\|_{L^{2}}^{2}+\left\|\partial_{y y} \theta(t)\right\|_{L^{2}}^{2}\right)+\mu_{2}\left\|\partial_{x x y} u_{1}\right\|_{L^{2}}^{2}+\mu_{2}\left\|\partial_{x y y} u_{1}\right\|_{L^{2}}^{2} \\
& \quad+\mu_{2}\left\|\partial_{y y y} u_{1}\right\|_{L^{2}}^{2}+\kappa_{1}\left\|\partial_{x x x} \theta\right\|_{L^{2}}^{2}+\kappa_{1}\left\|\partial_{x y y} \theta\right\|_{L^{2}}^{2}
\end{aligned}
$$




$$
\begin{aligned}
\leq & C\left(\left\|\partial_{y} u_{1}\right\|_{L^{2}}^{2}+\|\omega\|_{L^{2}}^{2}+\left\|\partial_{x} \theta\right\|_{L^{2}}^{2}+\left\|\partial_{y} \theta\right\|_{L^{2}}^{2}+\left\|u_{1}\right\|_{L^{2}}^{2}\left\|\partial_{y} u_{1}\right\|_{L^{2}}^{2}+\|\omega\|_{L^{2}}^{\frac{2}{3}}\left\|\partial_{x y} u_{1}\right\|_{L^{2}}^{\frac{2}{3}}\right. \\
& \left.+\left\|u_{2}\right\|_{L^{2}}^{2}\|\omega\|_{L^{2}}^{2}+\left\|\partial_{x x} u_{2}\right\|_{L^{4}}+1\right)\left(\|\nabla \omega\|_{L^{2}}^{2}+\left\|\partial_{x x} \theta\right\|_{L^{2}}^{2}+\left\|\partial_{y y} \theta\right\|_{L^{2}}^{2}\right) \\
& +C\left\|\partial_{x x} u_{2}\right\|_{L^{4}}\left(\left\|\partial_{y} u_{1}\right\|_{L^{2}}^{2}+\|\omega\|_{L^{2}}^{2}\right)
\end{aligned}
$$

It thus follows from Gronwall's inequality that

$$
\begin{aligned}
& \|\nabla \omega\|_{L^{2}}^{2}+\left\|\partial_{x x} \theta\right\|_{L^{2}}^{2}+\left\|\partial_{y y} \theta\right\|_{L^{2}}^{2}+\mu_{2} \int_{0}^{t}\left\|\partial_{x x y} u_{1}(\tau)\right\|_{L^{2}}^{2} d \tau+\mu_{2} \int_{0}^{t}\left\|\partial_{x y y} u_{1}(\tau)\right\|_{L^{2}}^{2} d \tau \\
& \quad+\mu_{2} \int_{0}^{t}\left\|\partial_{y y y} u_{1}(\tau)\right\|_{L^{2}}^{2} d \tau+\kappa_{1} \int_{0}^{t}\left\|\partial_{x x x} \theta(\tau)\right\|_{L^{2}}^{2} d \tau+\kappa_{1} \int_{0}^{t}\left\|\partial_{x y y} \theta(\tau)\right\|_{L^{2}}^{2} d \tau \\
& \quad \leq C
\end{aligned}
$$

which yields

$$
\begin{gathered}
\|\nabla \omega\|_{L^{\infty}\left([0, T) ; L^{2}\left(\mathbb{R}^{2}\right)\right)}+\|\Delta \theta\|_{L^{\infty}\left([0, T) ; L^{2}\left(\mathbb{R}^{2}\right)\right)}+\mu_{2}\left\|\Delta \partial_{y} u_{1}\right\|_{L^{2}\left([0, T) ; L^{2}\left(\mathbb{R}^{2}\right)\right)} \\
+\mu_{2}\left\|\partial_{x y y} u_{1}\right\|_{L^{2}\left([0, T) ; L^{2}\left(\mathbb{R}^{2}\right)\right)}+\kappa_{1}\left\|\Delta \partial_{x} \theta\right\|_{L^{2}\left([0, T) ; L^{2}\left(\mathbb{R}^{2}\right)\right)} \leq C .
\end{gathered}
$$

Therefore, we have completed the proof of Theorem 3.1.

Furthermore, we establish another regularity criterion to the 2D Bénard system with vertical dissipation and horizontal thermal diffusivity.

Theorem 3.2 Let $\mu_{1}=0, \mu_{2}>0, \mu_{3}=0, \mu_{4}=0, \kappa_{1}>0, \kappa_{2}=0$. Given a positive time $T \in$ $(0, \infty)$. Assume that $u_{1}^{0}, u_{2}^{0}, \theta^{0} \in H^{2}\left(\mathbb{R}^{2}\right)$ and $\nabla \cdot u^{0}=0$. Let $\left(u_{1}, u_{2}, \theta\right)$ be the solution of (1.2)-(1.3). If

$$
\int_{0}^{T}\left\|\partial_{x} u\right\|_{L^{\infty}}^{2} d t<\infty
$$

for some $T>0$, then $\left\|\left(u_{1}, u_{2}, \theta\right)\right\|_{H^{2}}$ is finite on $[0, T]$.

We now give the proof of Theorem 3.2, we first prove the global $H^{1}$ bound for $\left(u_{1}, u_{2}, \theta\right)$, we present the proof of the main theorem secondly.

- Global $H^{1}$-bound for $\left(u_{1}, u_{2}, \theta\right)$

Proposition 3.3 Let $\left(u_{1}^{0}, u_{2}^{0}, \theta^{0}\right) \in H^{2}\left(\mathbb{R}^{2}\right)$ and let $\left(u_{1}, u_{2}, \theta\right)$ be the corresponding solution of (1.2)-(1.3). Then $\left(u_{1}, u_{2}, \theta\right)$ obeys the following global $L^{2}$-bound:

$$
\begin{aligned}
& \left\|u_{1}\right\|_{L^{2}}^{2}+\left\|u_{2}\right\|_{L^{2}}^{2}+\|\theta\|_{L^{2}}^{2}+\mu_{2} \int_{0}^{t}\left\|\partial_{y} u_{1}(\tau)\right\|_{L^{2}}^{2} d \tau+\kappa_{1} \int_{0}^{t}\left\|\partial_{x} \theta(\tau)\right\|_{L^{2}}^{2} d \tau \\
& \quad \leq C\left\|\left(u_{0}^{1}, u_{2}^{0}, \theta^{0}\right)\right\|_{L^{2}}^{2}
\end{aligned}
$$

for any $t \geq 0$. Here $C$ is a constant depending only on $\mu_{2}, \kappa_{1}$, and $T$. 
Proposition 3.4 Assume that $\left(u_{1}^{0}, u_{2}^{0}, \theta^{0}\right) \in H^{2}\left(\mathbb{R}^{2}\right), \nabla \cdot u^{0}=0$. Let $\left(u_{1}, u_{2}, \theta\right)$ be the corresponding solution of (1.2)-(1.3). Then, for any $T>0$ and $t \leq T$,

$$
\left\|\left(u_{1}(t), u_{2}(t), \theta(t)\right)\right\|_{H^{1}\left(\mathbb{R}^{2}\right)} \leq C_{1} e^{C_{2} \int_{0}^{t}\left\|\partial_{x} u\right\|_{L^{\infty}}^{2} d \tau}
$$

where $C_{1}$ is a constant depending on $T$ and initial data, and $C_{2}$ is a pure constant.

Proof Taking the inner product of the first three equations in (1.2) with $-\Delta u_{1},-\Delta u_{2}$ and $-\Delta \theta$, respectively, integrating with respect to space

$$
\begin{gathered}
\frac{1}{2} \frac{d}{d t}\left(\left\|\nabla u_{1}(t)\right\|_{L^{2}}^{2}+\left\|\nabla u_{2}(t)\right\|_{L^{2}}^{2}+\|\nabla \theta(t)\|_{L^{2}}^{2}\right)+\mu_{2}\left\|\nabla \partial_{y} u_{1}\right\|_{L^{2}}^{2}+\kappa_{1}\left\|\nabla \partial_{x} \theta\right\|_{L^{2}}^{2} \\
=\underbrace{-\iint_{\mathbb{R}^{2}} \theta \cdot \Delta u_{2} d x d y}_{K_{1}}+\underbrace{\iint_{\mathbb{R}^{2}}[(u \cdot \nabla) \theta] \cdot \Delta \theta d x d y}_{K_{2}}-\int \underbrace{}_{\mathbb{R}^{2}} u_{2} \cdot \Delta \theta d x d y
\end{gathered}
$$

then, for notational convenience, we set

$$
A(t)=\left\|\nabla u_{1}(\cdot, t)\right\|_{L^{2}}^{2}+\left\|\nabla u_{2}(\cdot, t)\right\|_{L^{2}}^{2}+\|\nabla \theta(\cdot, t)\|_{L^{2}}^{2} .
$$

For $K_{1}$ and $K_{3}$, integrating by parts and applying the Hölder inequality gives

$$
K_{1}+K_{3} \leq\|\nabla u\|_{L^{2}}^{2}+\|\nabla \theta\|_{L^{2}}^{2}
$$

To estimate $K_{2}$ we write component-wise

$$
\begin{aligned}
K_{2}= & -\iint_{\mathbb{R}^{2}} \nabla \theta \cdot \nabla u \cdot \nabla \theta d x d y \\
= & \underbrace{-\iint_{\mathbb{R}^{2}} \partial_{x} \theta \partial_{x} u_{1} \partial_{x} \theta d x d y}_{K_{21}}-\underbrace{\iint_{\mathbb{R}^{2}} \partial_{x} \theta \partial_{x} u_{2} \partial_{y} \theta d x d y}_{K_{22}}-\underbrace{\iint_{\mathbb{R}^{2}} \partial_{y} \theta \partial_{y} u_{1} \partial_{x} \theta d x d y}_{K_{24}} \\
& -\iint_{\mathbb{R}^{2}} \partial_{y} \theta \partial_{y} u_{2} \partial_{y} \theta d x d y \\
K_{21}= & 2 \iint_{\mathbb{R}^{2}} u_{1} \partial_{x} \theta \partial_{x x} \theta d x d y \\
\leq & C\left\|\partial_{x x} \theta\right\|_{L^{2}}\left\|u_{1}\right\|_{L^{2}}^{\frac{1}{2}}\left\|\partial_{y} u_{1}\right\|_{L^{2}}^{\frac{1}{2}}\left\|\partial_{x} \theta\right\|_{L^{2}}^{\frac{1}{2}}\left\|\partial_{x x} \theta\right\|_{L^{2}}^{\frac{1}{2}} \\
\leq & \frac{\kappa_{1}}{6}\left\|\nabla \partial_{x} \theta\right\|_{L^{2}}^{2}+C\left\|u_{1}\right\|_{L^{2}}^{2}\left\|\partial_{y} u_{1}\right\|_{L^{2}}^{2}\|\nabla \theta\|_{L^{2}}^{2}, \\
K_{22} \leq & C\left\|\partial_{x} u_{2}\right\|_{L^{\infty}}\|\nabla \theta\|_{L^{2}}^{2}, \\
K_{23} \leq & C\left\|\partial_{y} u_{1}\right\|_{L^{2}}\left\|\partial_{y} \theta\right\|_{L^{2}}^{\frac{1}{2}}\left\|\partial_{x y} \theta\right\|_{L^{2}}^{\frac{1}{2}}\left\|\partial_{x} \theta\right\|_{L^{2}}^{\frac{1}{2}}\left\|\partial_{x y} \theta\right\|_{L^{2}}^{\frac{1}{2}} \\
\leq & \frac{\kappa_{1}}{6}\left\|\nabla \partial_{x} \theta\right\|_{L^{2}}^{2}+C\left\|\partial_{y} u_{1}\right\|_{L^{2}}^{2}\|\nabla \theta\|_{L^{2}}^{2},
\end{aligned}
$$




$$
\begin{aligned}
K_{24} & =-2 \iint_{\mathbb{R}^{2}} u_{1} \partial_{y} \theta \partial_{x y} \theta d x d y \\
& \leq C\left\|\partial_{x y} \theta\right\|_{L^{2}}\left\|u_{1}\right\|_{L^{2}}^{\frac{1}{2}}\left\|\partial_{y} u_{1}\right\|_{L^{2}}^{\frac{1}{2}}\left\|\partial_{y} \theta\right\|_{L^{2}}^{\frac{1}{2}}\left\|\partial_{x y} \theta\right\|_{L^{2}}^{\frac{1}{2}} \\
& \leq \frac{\kappa_{1}}{6}\left\|\nabla \partial_{x} \theta\right\|_{L^{2}}^{2}+C\left\|u_{1}\right\|_{L^{2}}^{2}\left\|\partial_{y} u_{1}\right\|_{L^{2}}^{2}\|\nabla \theta\|_{L^{2}}^{2} .
\end{aligned}
$$

After combining inequalities,

$$
\begin{aligned}
\frac{1}{2} \frac{d}{d t} A(t)+\mu_{2}\left\|\nabla \partial_{y} u_{1}\right\|_{L^{2}}^{2}+\kappa_{1}\left\|\nabla \partial_{x} \theta\right\|_{L^{2}}^{2} \leq & \frac{\mu_{2}}{2}\left\|\nabla \partial_{y} u_{1}\right\|_{L^{2}}^{2}+\frac{\kappa_{1}}{2}\left\|\nabla \partial_{x} \theta\right\|_{L^{2}}^{2} \\
& +C\left\|\partial_{x} u_{2}\right\|_{L^{\infty}}^{2} A(t)
\end{aligned}
$$

after applying Gronwall's lemma, we get the $H^{1}$-norm for $u_{1}, u_{2}$, and $\theta$. This completes the proof of Proposition 3.4.

- $H^{2}$ bound

To estimate the $H^{2}$-norm of $\left(u_{1}, u_{2}, \theta\right)$, we consider the equation of $\omega=\nabla \times u, \nabla \theta$

$$
\left\{\begin{array}{l}
\partial_{t} \omega+(u \cdot \nabla) \omega=-\mu_{2} \partial_{y y y} u_{1}+\partial_{x} \theta \\
\partial_{t} \nabla \theta+\nabla[(u \cdot \nabla) \theta]=\kappa_{1} \nabla \partial_{x x} \theta+\nabla u_{2}
\end{array}\right.
$$

Proposition 3.5 Assume that $\left(u_{1}^{0}, u_{2}^{0}, \theta^{0}\right) \in H^{2}\left(\mathbb{R}^{2}\right), \nabla \cdot u^{0}=0$ and let $\left(u_{1}, u_{2}, \theta\right)$ be the solution of (1.2)-(1.3). Then $(\omega, \nabla \theta)$ satisfy

$$
\|\omega\|_{L^{2}}^{2}+\|\Delta \theta\|_{L^{2}}^{2}+\mu_{2} \int_{0}^{T}\left\|\Delta \partial_{y} u_{1}\right\|_{L^{2}}^{2} d t+\kappa_{1} \int_{0}^{T}\left\|\Delta \partial_{x} \theta\right\|_{L^{2}}^{2} d t \leq C
$$

if $\partial_{x} u \in L^{2}\left([0, T] ; L^{\infty}\left(\mathbb{R}^{2}\right)\right)$.

Proof Taking the inner product of $(3.38)_{1}$ with $-\Delta \omega$ and $(3.38)_{2}$ with $-\Delta \nabla \theta$ in $L^{2}\left(\mathbb{R}^{2}\right)$, respectively, we find

$$
\begin{gathered}
\frac{1}{2} \frac{d}{d t}\left(\|\nabla \omega\|_{L^{2}}^{2}+\|\Delta \theta\|_{L^{2}}^{2}\right)+\mu_{2}\left\|\Delta \partial_{y} u_{1}\right\|_{L^{2}}^{2}+\kappa_{1}\left\|\Delta \partial_{x} \theta\right\|_{L^{2}}^{2} \\
=\underbrace{\iint_{\mathbb{R}^{2}}(u \cdot \nabla) \omega \cdot \Delta \omega d x d y}_{L_{1}}+\int \underbrace{\iint_{\mathbb{R}^{2}} \nabla \partial_{x} \theta \cdot \nabla \omega d x d y}_{L_{3}} \\
\underbrace{-\iint_{\mathbb{R}^{2}} \Delta[(u \cdot \nabla) \theta] \Delta \theta d x d y}_{L_{2}}+\underbrace{\iint_{\int^{2}} \Delta \theta}_{L_{\mathbb{R}^{2}} \Delta u_{2} \cdot \Delta \theta d x d y} .
\end{gathered}
$$

We now estimate $L_{1}$ through $L_{4}$. We firstly write the four terms in $L_{1}$ explicitly,

$$
\begin{aligned}
L_{1}= & -\iint_{\mathbb{R}^{2}} \nabla \omega \cdot \nabla u \cdot \nabla \omega d x d y \\
= & -\underbrace{-\iint_{\mathbb{R}^{2}} \partial_{x} u_{1} \partial_{x} \omega \partial_{x} \omega d x d y}_{L_{11}} \underbrace{-\iint_{\mathbb{R}^{2}} \partial_{x} u_{2} \partial_{x} \omega \partial_{y} \omega d x d y}_{L_{12}} \\
& \underbrace{-\iint_{\mathbb{R}^{2}} \partial_{y} u_{1} \partial_{x} \omega \partial_{y} \omega d x d y}_{L_{13}} \underbrace{-\iint_{\mathbb{R}^{2}} \partial_{y} u_{2} \partial_{y} \omega \partial_{y} \omega d x d y}_{L_{14}} .
\end{aligned}
$$


These terms can be bounded as follows:

$$
\begin{aligned}
L_{11} & \leq\left\|\partial_{x} u_{1}\right\|_{L^{\infty}}\left\|\partial_{x} \omega\right\|_{L^{2}}^{2} \leq\left\|\partial_{x} u_{1}\right\|_{L^{\infty}}\|\nabla \omega\|_{L^{2}}^{2}, \\
L_{12} & \leq\left\|\partial_{x} u_{2}\right\|_{L^{\infty}}\left\|\partial_{x} \omega\right\|_{L^{2}}\left\|\partial_{y} \omega\right\|_{L^{2}} \leq\left\|\partial_{x} u_{2}\right\|_{L^{\infty}}\|\nabla \omega\|_{L^{2}}^{2}, \\
L_{13} & \leq C\left\|\partial_{x} \omega\right\|_{L^{2}}\left\|\partial_{y} u_{1}\right\|_{L^{2}}^{\frac{1}{2}}\left\|\partial_{x y} u_{1}\right\|_{L^{2}}^{\frac{1}{2}}\left\|\partial_{y} \omega\right\|_{L^{2}}^{\frac{1}{2}}\left\|\partial_{y y} \omega\right\|_{L^{2}}^{\frac{1}{2}} \\
& \leq \frac{\mu_{2}}{6}\left\|\Delta \partial_{y} u_{1}\right\|_{L^{2}}^{2}+C\left\|\partial_{y} u_{1}\right\|_{L^{2}}^{\frac{2}{3}}\left\|\nabla \partial_{y} u_{1}\right\|_{L^{2}}^{\frac{2}{3}}\|\nabla \omega\|_{L^{2}}^{2}, \\
L_{14} & =2 \iint_{\mathbb{R}^{2}} u_{2} \partial_{y} \omega \partial_{y y} \omega d x d y \\
& \leq C\left\|\partial_{y y} \omega\right\|_{L^{2}}\left\|u_{2}\right\|_{L^{2}}^{\frac{1}{2}}\left\|\partial_{x} u_{2}\right\|_{L^{2}}^{\frac{1}{2}}\left\|\partial_{y} \omega\right\|_{L^{2}}^{\frac{1}{2}}\left\|\partial_{y y} \omega\right\|_{L^{2}}^{\frac{1}{2}} \\
& \leq \frac{\mu_{2}}{6}\left\|\Delta \partial_{y} u_{1}\right\|_{L^{2}}^{2}+C\left\|u_{2}\right\|_{L^{2}}^{2}\|\nabla u\|_{L^{2}}^{2}\|\nabla \omega\|_{L^{2}}^{2} .
\end{aligned}
$$

The terms $L_{2}$ and $L_{4}$ can easily be bounded,

$$
\begin{aligned}
& L_{2} \leq \underbrace{\|\nabla \omega\|_{L^{2}}^{2}+\|\Delta \theta\|_{L^{2}}^{2}}_{\left\|\nabla \partial_{x} \theta\right\|_{L^{2}} \leq\|\Delta \theta\|_{L^{2}}}, \\
& L_{4} \leq \underbrace{\|\nabla \omega\|_{L^{2}}^{2}+\|\Delta \theta\|_{L^{2}}^{2}}_{\left(\left\|\Delta u_{2}\right\|_{L^{2}} \leq\|\nabla \omega\|_{L^{2}}\right)} .
\end{aligned}
$$

Finally, we deal with $L_{3}$.

$$
L_{3}=-\iint_{\mathbb{R}^{2}} \Delta\left(u_{1} \partial_{x} \theta+u_{2} \partial_{y} \theta\right) \Delta \theta d x d y \triangleq L_{31}+L_{32} .
$$

We first split $L_{31}$ and $L_{32}$ each into two terms,

$$
\begin{aligned}
& L_{31}=-\iint_{\mathbb{R}^{2}} \partial_{x x}\left(u_{1} \partial_{x} \theta+u_{2} \partial_{y} \theta\right) \Delta \theta d x d y \triangleq L_{311}+L_{312}, \\
& L_{32}=-\iint_{\mathbb{R}^{2}} \partial_{y y}\left(u_{1} \partial_{x} \theta+u_{2} \partial_{y} \theta\right) \partial_{x x} \theta d x d y-\iint_{\mathbb{R}^{2}} \partial_{y y}\left(u_{1} \partial_{x} \theta+u_{2} \partial_{y} \theta\right) \partial_{y y} \theta d x d y \\
& \quad \triangleq L_{321}+L_{322} .
\end{aligned}
$$

These terms are bounded as follows:

$$
\begin{aligned}
L_{311}= & -\iint_{\mathbb{R}^{2}} \partial_{x x}\left(u_{1} \partial_{x} \theta\right) \Delta \theta d x d y \\
= & \iint_{\mathbb{R}^{2}} \partial_{x} u_{1} \partial_{x} \theta \Delta \partial_{x} \theta d x d y+\iint_{\mathbb{R}^{2}} u_{1} \partial_{x x} \theta \Delta \partial_{x} \theta d x d y \\
\leq & C\left\|\Delta \partial_{x} \theta\right\|_{L^{2}}\left\|\partial_{x} u_{1}\right\|_{L^{2}}^{\frac{1}{2}}\left\|\partial_{x y} u_{1}\right\|_{L^{2}}^{\frac{1}{2}}\left\|\partial_{x} \theta\right\|_{L^{2}}^{\frac{1}{2}}\left\|\partial_{x x} \theta\right\|_{L^{2}}^{\frac{1}{2}} \\
& +C\left\|\Delta \partial_{x} \theta\right\|_{L^{2}}\left\|u_{1}\right\|_{L^{2}}^{\frac{1}{2}}\left\|\partial_{y} u_{1}\right\|_{L^{2}}^{\frac{1}{2}}\left\|\partial_{x x} \theta\right\|_{L^{2}}^{\frac{1}{2}}\left\|\partial_{x x x} \theta\right\|_{L^{2}}^{\frac{1}{2}} \\
\leq & \frac{\kappa_{1}}{18}\left\|\Delta \partial_{x} \theta\right\|_{L^{2}}^{2}+C\|\nabla u\|_{L^{2}}^{2}\|\nabla \omega\|_{L^{2}}^{2} \\
& +C\left(\|\nabla \theta\|_{L^{2}}^{2}+\left\|u_{1}\right\|_{L^{2}}^{2}\left\|\partial_{y} u_{1}\right\|_{L^{2}}^{2}\right)\|\Delta \theta\|_{L^{2}}^{2},
\end{aligned}
$$


Ma and Zhang Boundary Value Problems （2018) 2018:79

Page 18 of 23

$$
\begin{aligned}
& L_{312}=-\iint_{\mathbb{R}^{2}} \partial_{x x}\left(u_{2} \partial_{y} \theta\right) \Delta \theta d x d y \\
& =\iint_{\mathbb{R}^{2}} \partial_{x} u_{2} \partial_{y} \theta \Delta \partial_{x} \theta d x d y+\iint_{\mathbb{R}^{2}} u_{2} \partial_{x y} \theta \Delta \partial_{x} \theta d x d y \\
& \leq\left\|\partial_{x} u_{2}\right\|_{L^{\infty}}\left\|\partial_{y} \theta\right\|_{L^{2}}\left\|\Delta \partial_{x} \theta\right\|_{L^{2}} \\
& +C\left\|\Delta \partial_{x} \theta\right\|_{L^{2}}\left\|u_{2}\right\|_{L^{2}}^{\frac{1}{2}}\left\|\partial_{x} u_{2}\right\|_{L^{2}}^{\frac{1}{2}}\left\|\partial_{x y} \theta\right\|_{L^{2}}^{\frac{1}{2}}\left\|\partial_{x y y} \theta\right\|_{L^{2}}^{\frac{1}{2}} \\
& \leq \frac{\kappa_{1}}{18}\left\|\Delta \partial_{x} \theta\right\|_{L^{2}}^{2}+C\left\|\partial_{x} u_{2}\right\|_{L^{\infty}}^{2}\|\nabla \theta\|_{L^{2}}^{2}+C\left\|u_{2}\right\|_{L^{2}}^{2}\|\nabla u\|_{L^{2}}^{2}\left\|\nabla \partial_{x} \theta\right\|_{L^{2}}^{2} \text {, } \\
& L_{321}=-\iint_{\mathbb{R}^{2}} \partial_{y y}\left(u_{1} \partial_{x} \theta+u_{2} \partial_{y} \theta\right) \partial_{x x} \theta d x d y \\
& =\iint_{\mathbb{R}^{2}} \partial_{x} u_{1} \partial_{x} \theta \partial_{x y y} \theta d x d y+\iint_{\mathbb{R}^{2}} u_{1} \partial_{x x} \theta \partial_{x y y} \theta d x d y \\
& +\iint_{\mathbb{R}^{2}} \partial_{x} u_{2} \partial_{y} \theta \partial_{x y y} \theta d x d y+\iint_{\mathbb{R}^{2}} u_{2} \partial_{x y} \theta \partial_{x y y} \theta d x d y \\
& \leq \frac{\kappa_{1}}{18}\left\|\Delta \partial_{x} \theta\right\|_{L^{2}}^{2}+C\left\|\partial_{x} u\right\|_{L^{\infty}}^{2}\|\nabla \theta\|_{L^{2}}^{2}+C\left\|u_{1}\right\|_{L^{2}}^{2}\|\nabla u\|_{L^{2}}^{2}\|\Delta \theta\|_{L^{2}}^{2} \\
& +C\left\|u_{2}\right\|_{L^{2}}^{2}\|\nabla u\|_{L^{2}}^{2}\left\|\nabla \partial_{x} \theta\right\|_{L^{2}}^{2}, \\
& L_{322}=-\iint_{\mathbb{R}^{2}} \partial_{y y}\left(u_{1} \partial_{x} \theta+u_{2} \partial_{y} \theta\right) \partial_{y y} \theta d x d y \\
& =-\iint_{\mathbb{R}^{2}} \partial_{y}\left(\partial_{y} u_{1} \partial_{x} \theta+u_{1} \partial_{x y} \theta\right) \partial_{y y} \theta d x d y \\
& -\iint_{\mathbb{R}^{2}} \partial_{y}\left(\partial_{y} u_{2} \partial_{y} \theta+u_{2} \partial_{y y} \theta\right) \partial_{y y} \theta d x d y \\
& =-\iint_{\mathbb{R}^{2}}\left(\partial_{y y} u_{1} \partial_{x} \theta+2 \partial_{y} u_{1} \partial_{x y} \theta+u_{1} \partial_{x y y} \theta\right) \partial_{y y} \theta d x d y \\
& -\iint_{\mathbb{R}^{2}}\left(\partial_{y y} u_{2} \partial_{y} \theta+2 \partial_{y} u_{2} \partial_{y y} \theta+u_{2} \partial_{y y y} \theta\right) \partial_{y y} \theta d x d y, \\
& \left|-\iint_{\mathbb{R}^{2}} \partial_{y y} u_{1} \partial_{x} \theta \partial_{y y} \theta d x d y\right| \\
& \leq C\left\|\partial_{x} \theta\right\|_{L^{2}}\left\|\partial_{y y} u_{1}\right\|_{L^{2}}^{\frac{1}{2}}\left\|\partial_{y y y} u_{1}\right\|_{L^{2}}^{\frac{1}{2}}\left\|\partial_{y y} \theta\right\|_{L^{2}}^{\frac{1}{2}}\left\|\partial_{x y y} \theta\right\|_{L^{2}}^{\frac{1}{2}} \\
& \leq \frac{\mu_{2}}{6}\left\|\Delta \partial_{y} u_{1}\right\|_{L^{2}}^{2}+\frac{\kappa_{1}}{18}\left\|\Delta \partial_{x} \theta\right\|_{L^{2}}^{2}+C\left\|\partial_{x} \theta\right\|_{L^{2}}^{2}\left(\|\nabla \omega\|_{L^{2}}^{2}+\|\Delta \theta\|_{L^{2}}^{2}\right) \text {, } \\
& \left|-2 \iint_{\mathbb{R}^{2}} \partial_{y} u_{1} \partial_{x y} \theta \partial_{y y} \theta d x d y\right| \\
& \leq C\left\|\partial_{y y} \theta\right\|_{L^{2}}\left\|\partial_{y} u_{1}\right\|_{L^{2}}^{\frac{1}{2}}\left\|\partial_{x y} u_{1}\right\|_{L^{2}}^{\frac{1}{2}}\left\|\partial_{x y} \theta\right\|_{L^{2}}^{\frac{1}{2}}\left\|\partial_{x y y} \theta\right\|_{L^{2}}^{\frac{1}{2}} \\
& \leq \frac{\kappa_{1}}{18}\left\|\Delta \partial_{x} \theta\right\|_{L^{2}}^{2}+C\left\|\partial_{y} u_{1}\right\|_{L^{2}}^{2}\|\nabla \omega\|_{L^{2}}^{2}+C\left\|\nabla \partial_{x} \theta\right\|_{L^{2}}\|\Delta \theta\|_{L^{2}}^{2} \text {, } \\
& \left|-\iint_{\mathbb{R}^{2}} u_{1} \partial_{x y y} \theta \partial_{y y} \theta d x d y\right| \\
& \leq C\left\|\partial_{x y y} \theta\right\|_{L^{2}}\left\|u_{1}\right\|_{L^{2}}^{\frac{1}{2}}\left\|\partial_{y} u_{1}\right\|_{L^{2}}^{\frac{1}{2}}\left\|\partial_{y y} \theta\right\|_{L^{2}}^{\frac{1}{2}}\left\|\partial_{x y y} \theta\right\|_{L^{2}}^{\frac{1}{2}} \\
& \leq \frac{\kappa_{1}}{18}\left\|\Delta \partial_{x} \theta\right\|_{L^{2}}^{2}+C\left\|u_{1}\right\|_{L^{2}}^{2}\left\|\partial_{y} u_{1}\right\|_{L^{2}}^{2}\|\Delta \theta\|_{L^{2}}^{2} \text {, }
\end{aligned}
$$




$$
\begin{aligned}
& \left|-\iint_{\mathbb{R}^{2}} \partial_{y y} u_{2} \partial_{y} \theta \partial_{y y} \theta d x d y\right| \\
& \quad \leq C\left\|\partial_{y y} u_{2}\right\|_{L^{2}}\left\|\partial_{y} \theta\right\|_{L^{2}}^{\frac{1}{2}}\left\|\partial_{y y} \theta\right\|_{L^{2}}^{\frac{1}{2}}\left\|\partial_{y y} \theta\right\|_{L^{2}}^{\frac{1}{2}}\left\|\partial_{x y y} \theta\right\|_{L^{2}}^{\frac{1}{2}} \\
& \quad \leq \frac{\kappa_{1}}{18}\left\|\Delta \partial_{x} \theta\right\|_{L^{2}}^{2}+C\|\nabla \theta\|_{L^{2}}^{2}+C\left\|\nabla \partial_{y} u_{1}\right\|_{L^{2}}^{2}\|\Delta \theta\|_{L^{2}}^{2}, \\
& \quad-2 \iint_{\mathbb{R}^{2}} \partial_{y} u_{2} \partial_{y y} \theta \partial_{y y} \theta d x d y \mid \\
& \quad=\left|-4 \iint_{\mathbb{R}^{2}} u_{1} \partial_{y y} \theta \partial_{x y y} \theta d x d y\right| \\
& \quad \leq C\left\|\partial_{x y y} \theta\right\|_{L^{2}}\left\|u_{1}\right\|_{L^{2}}^{\frac{1}{2}}\left\|\partial_{y} u_{1}\right\|_{L^{2}}^{\frac{1}{2}}\left\|\partial_{y y} \theta\right\|_{L^{2}}^{\frac{1}{2}}\left\|\partial_{x y y} \theta\right\|_{L^{2}}^{\frac{1}{2}} \\
& \quad \leq \frac{\kappa_{1}}{18}\left\|\Delta \partial_{x} \theta\right\|_{L^{2}}^{2}+C\left\|u_{1}\right\|_{L^{2}}^{2}\left\|\partial_{y} u_{1}\right\|_{L^{2}}^{2}\|\Delta \theta\|_{L^{2}}^{2}, \\
& -\iint_{\mathbb{R}^{2}} u_{2} \partial_{y y y} \theta \partial_{y y} \theta d x d y \mid \\
& \quad=\left|\iint_{\mathbb{R}^{2}} u_{1} \partial_{y y} \theta \partial_{x y y} \theta d x d y\right| \\
& \quad \leq \frac{\kappa_{1}}{18}\left\|\Delta \partial_{x} \theta\right\|_{L^{2}}^{2}+C\left\|u_{1}\right\|_{L^{2}}^{2}\left\|\partial_{y} u_{1}\right\|_{L^{2}}^{2}\|\Delta \theta\|_{L^{2}}^{2} .
\end{aligned}
$$

After combining all inequalities, together with Gronwall's inequality, we obtain the $H^{1}$ bound for $\omega, \nabla \theta$. Therefore, we obtain the global $H^{2}$ bound for $(u, \theta)$ for the 2D Bénard system (1.2)-(1.3) with vertical dissipation in the horizontal velocity equation and horizontal dissipation in the temperature equation. We thus complete the proof of Theorem 3.2.

\section{Global existence and regularity criteria of weak solution for Bénard system with other partial dissipation}

We devote this section to showing the global weak solution and regularity criteria for the 2D Bénard system with other cases for partial viscosity and thermal diffusivity. More precisely, we shall show the following cases and theorems.

Theorem 4.1 Let (i) $\mu_{1}=0, \mu_{2}=0, \mu_{3}>0, \mu_{4}=0, \kappa_{1}=0, \kappa_{2}>0$; (ii) $\mu_{1}=0, \mu_{2}=0, \mu_{3}>0$, $\mu_{4}=0, \kappa_{1}>0, \kappa_{2}=0$. Suppose that $u_{1}^{0}, u_{2}^{0}, \theta^{0} \in H^{1}\left(\mathbb{R}^{2}\right)$ and $\nabla \cdot u^{0}=0$. Then the problem (1.2)-(1.3) with (i) and (ii) admits a global weak solution $\left(u_{1}, u_{2}, \theta\right)$, which obeys

$$
\begin{array}{ll}
u_{1}, u_{2}, \theta \in L^{\infty}\left([0, T) ; H^{1}\left(\mathbb{R}^{2}\right)\right), & \partial_{x} u_{2}, \partial_{y} \theta \in L^{2}\left([0, T) ; H^{1}\left(\mathbb{R}^{2}\right)\right) ; \\
u_{1}, u_{2}, \theta \in L^{\infty}\left([0, T) ; H^{1}\left(\mathbb{R}^{2}\right)\right), & \partial_{x} u_{2}, \partial_{x} \theta \in L^{2}\left([0, T) ; H^{1}\left(\mathbb{R}^{2}\right)\right)
\end{array}
$$

for any $T>0$, respectively. Moreover, suppose that $u_{1}^{0}, u_{2}^{0}, \theta^{0} \in H^{2}\left(\mathbb{R}^{2}\right)$ and $\nabla \cdot u^{0}=0$. If one of the following two conditions holds:

$$
\begin{aligned}
& \int_{0}^{T}\left\|\partial_{y y} u_{1}(\tau)\right\|_{L^{4}\left(\mathbb{R}^{2}\right)} d \tau<\infty \\
& \int_{0}^{T}\left\|\partial_{y} u_{1}(\tau)\right\|_{L^{\infty}\left(\mathbb{R}^{2}\right)} d \tau<\infty
\end{aligned}
$$


for any fixed $T>0$, then the problem (1.2)-(1.3) with (i) and (ii) admits a global classical solution $\left(u_{1}, u_{2}, \theta\right)$, which, respectively, obeys

$$
\begin{aligned}
& u_{1}, u_{2}, \theta \in L^{\infty}\left([0, T) ; H^{2}\left(\mathbb{R}^{2}\right)\right), \\
& \partial_{x x y} u_{2} \in L^{2}\left([0, T) ; L^{2}\left(\mathbb{R}^{2}\right)\right), \quad \partial_{x} u_{2}, \partial_{y} \theta \in L^{2}\left([0, T) ; H^{2}\left(\mathbb{R}^{2}\right)\right) ; \\
& u_{1}, u_{2}, \theta \in L^{\infty}\left([0, T) ; H^{2}\left(\mathbb{R}^{2}\right)\right), \\
& \partial_{x x y} u_{2} \in L^{2}\left([0, T) ; L^{2}\left(\mathbb{R}^{2}\right)\right), \quad \partial_{x} u_{2}, \partial_{x} \theta \in L^{2}\left([0, T) ; H^{2}\left(\mathbb{R}^{2}\right)\right) .
\end{aligned}
$$

Theorem 4.2 Let (iii) $\mu_{1}>0, \mu_{2}=0, \mu_{3}=0, \mu_{4}=0, \kappa_{1}>0, \kappa_{2}=0$; (iv) $\mu_{1}>0, \mu_{2}=0$, $\mu_{3}=0, \mu_{4}=0, \kappa_{1}=0, \kappa_{2}>0$. Suppose that $u_{1}^{0}, u_{2}^{0}, \theta^{0} \in H^{1}\left(\mathbb{R}^{2}\right)$ and $\nabla \cdot u^{0}=0$. Then the problem (1.2)-(1.3) with (iii) and (iv) admits a global weak solution $\left(u_{1}, u_{2}, \theta\right)$, which obeys

$$
\begin{array}{ll}
u_{1}, u_{2}, \theta \in L^{\infty}\left([0, T) ; H^{1}\left(\mathbb{R}^{2}\right)\right), & \partial_{x} u_{1}, \partial_{x} \theta \in L^{2}\left([0, T) ; H^{1}\left(\mathbb{R}^{2}\right)\right) ; \\
u_{1}, u_{2}, \theta \in L^{\infty}\left([0, T) ; H^{1}\left(\mathbb{R}^{2}\right)\right), & \partial_{x} u_{1}, \partial_{y} \theta \in L^{2}\left([0, T) ; H^{1}\left(\mathbb{R}^{2}\right)\right)
\end{array}
$$

for any $T>0$, respectively. Furthermore, suppose that $u_{1}^{0}, u_{2}^{0}, \theta^{0} \in H^{2}\left(\mathbb{R}^{2}\right)$ and $\nabla \cdot u^{0}=0$. If one of the following three conditions holds:

$$
\begin{aligned}
& \int_{0}^{T}\left\|\partial_{y y} u_{2}(\tau)\right\|_{L^{4}\left(\mathbb{R}^{2}\right)} d \tau<\infty \\
& \int_{0}^{T}\left\|\partial_{x y} u_{1}(\tau)\right\|_{L^{4}\left(\mathbb{R}^{2}\right)} d \tau<\infty ; \\
& \int_{0}^{T}\left\|\partial_{y} u_{2}(\tau)\right\|_{L^{\infty}\left(\mathbb{R}^{2}\right)} d \tau<\infty
\end{aligned}
$$

for any fixed $T>0$, then the problem (1.2)-(1.3) with (iii) and (iv) admits a global classical solution $\left(u_{1}, u_{2}, \theta\right)$, which, respectively, obeys

$$
\begin{aligned}
& u_{1}, u_{2}, \theta \in L^{\infty}\left([0, T) ; H^{2}\left(\mathbb{R}^{2}\right)\right), \\
& \partial_{x x y} u_{1} \in L^{2}\left([0, T) ; L^{2}\left(\mathbb{R}^{2}\right)\right), \quad \partial_{x} u_{1}, \partial_{x} \theta \in L^{2}\left([0, T) ; H^{2}\left(\mathbb{R}^{2}\right)\right) ; \\
& u_{1}, u_{2}, \theta \in L^{\infty}\left([0, T) ; H^{2}\left(\mathbb{R}^{2}\right)\right), \\
& \partial_{x x y} u_{1} \in L^{2}\left([0, T) ; L^{2}\left(\mathbb{R}^{2}\right)\right), \quad \partial_{x} u_{1}, \partial_{y} \theta \in L^{2}\left([0, T) ; H^{2}\left(\mathbb{R}^{2}\right)\right) .
\end{aligned}
$$

Theorem 4.3 Let (v) $\mu_{1}=0, \mu_{2}>0, \mu_{3}=0, \mu_{4}=0, \kappa_{1}=0, \kappa_{2}>0$. Suppose that $u_{1}^{0}, u_{2}^{0}, \theta^{0} \in$ $H^{1}\left(\mathbb{R}^{2}\right)$ and $\nabla \cdot u^{0}=0$. Then the problem (1.2)-(1.3) with (v) admits a global weak solution $\left(u_{1}, u_{2}, \theta\right)$, which obeys

$$
u_{1}, u_{2}, \theta \in L^{\infty}\left([0, T) ; H^{1}\left(\mathbb{R}^{2}\right)\right), \quad \partial_{y} u_{1}, \partial_{y} \theta \in L^{2}\left([0, T) ; H^{1}\left(\mathbb{R}^{2}\right)\right)
$$

for any $T>0$. Moreover, suppose that $u_{1}^{0}, u_{2}^{0}, \theta^{0} \in H^{2}\left(\mathbb{R}^{2}\right)$ and $\nabla \cdot u^{0}=0$. If one of the following two conditions holds:

$$
\begin{aligned}
& \int_{0}^{T}\left\|\partial_{x x} u_{2}(\tau)\right\|_{L^{4}\left(\mathbb{R}^{2}\right)} d \tau<\infty ; \\
& \int_{0}^{T}\left\|\partial_{x} u_{2}(\tau)\right\|_{L^{\infty}\left(\mathbb{R}^{2}\right)} d \tau<\infty
\end{aligned}
$$


for any fixed $T>0$, then the problem (1.2)-(1.3) with (v) admits a global classical solution $\left(u_{1}, u_{2}, \theta\right)$, which obeys

$$
\begin{aligned}
& u_{1}, u_{2}, \theta \in L^{\infty}\left([0, T) ; H^{2}\left(\mathbb{R}^{2}\right)\right), \\
& \partial_{x y y} u_{1} \in L^{2}\left([0, T) ; L^{2}\left(\mathbb{R}^{2}\right)\right), \quad \partial_{y} u_{1}, \partial_{y} \theta \in L^{2}\left([0, T) ; H^{2}\left(\mathbb{R}^{2}\right)\right) .
\end{aligned}
$$

Theorem 4.4 Let (vi) $\mu_{1}=0, \mu_{2}=0, \mu_{3}=0, \mu_{4}>0, \kappa_{1}>0, \kappa_{2}=0$ and (vii) $\mu_{1}=0, \mu_{2}=0$, $\mu_{3}=0, \mu_{4}>0, \kappa_{1}=0, \kappa_{2}>0$. Suppose that $u_{1}^{0}, u_{2}^{0}, \theta^{0} \in H^{1}\left(\mathbb{R}^{2}\right)$ and $\nabla \cdot u^{0}=0$. Then the problem (1.2)-(1.3) with (vi) and (vii) admits a global weak solution $\left(u_{1}, u_{2}, \theta\right)$, which obeys

$$
\begin{array}{ll}
u_{1}, u_{2}, \theta \in L^{\infty}\left([0, T) ; H^{1}\left(\mathbb{R}^{2}\right)\right), & \partial_{y} u_{2}, \partial_{x} \theta \in L^{2}\left([0, T) ; H^{1}\left(\mathbb{R}^{2}\right)\right) ; \\
u_{1}, u_{2}, \theta \in L^{\infty}\left([0, T) ; H^{1}\left(\mathbb{R}^{2}\right)\right), & \partial_{y} u_{2}, \partial_{y} \theta \in L^{2}\left([0, T) ; H^{1}\left(\mathbb{R}^{2}\right)\right)
\end{array}
$$

for any $T>0$, respectively. Furthermore, suppose that $u_{1}^{0}, u_{2}^{0}, \theta^{0} \in H^{2}\left(\mathbb{R}^{2}\right)$ and $\nabla \cdot u^{0}=0$. If one of the following three conditions holds:

$$
\begin{aligned}
& \int_{0}^{T}\left\|\partial_{x x} u_{1}(\tau)\right\|_{L^{4}\left(\mathbb{R}^{2}\right)} d \tau<\infty ; \\
& \int_{0}^{T}\left\|\partial_{x y} u_{2}(\tau)\right\|_{L^{4}\left(\mathbb{R}^{2}\right)} d \tau<\infty \\
& \int_{0}^{T}\left\|\partial_{x} u_{1}(\tau)\right\|_{L^{\infty}\left(\mathbb{R}^{2}\right)} d \tau<\infty
\end{aligned}
$$

for any fixed $T>0$, then the problem (1.2)-(1.3) admits a global classical solution $\left(u_{1}, u_{2}, \theta\right)$, which, respectively, obeys

$$
\begin{aligned}
& u_{1}, u_{2}, \theta \in L^{\infty}\left([0, T) ; H^{2}\left(\mathbb{R}^{2}\right)\right), \\
& \partial_{x y y} u_{2} \in L^{2}\left([0, T) ; L^{2}\left(\mathbb{R}^{2}\right)\right), \quad \partial_{y} u_{2}, \partial_{x} \theta \in L^{2}\left([0, T) ; H^{2}\left(\mathbb{R}^{2}\right)\right) ; \\
& u_{1}, u_{2}, \theta \in L^{\infty}\left([0, T) ; H^{2}\left(\mathbb{R}^{2}\right)\right), \\
& \partial_{x y y} u_{2} \in L^{2}\left([0, T) ; L^{2}\left(\mathbb{R}^{2}\right)\right), \quad \partial_{y} u_{2}, \partial_{y} \theta \in L^{2}\left([0, T) ; H^{2}\left(\mathbb{R}^{2}\right)\right) .
\end{aligned}
$$

Due to those theorems' proofs being similar to the results of Sect. 3, we can leave the proofs of those theorems to the interested readers.

\section{Conclusion}

The Bénard fluid problem is a very classical problem in the fluid dynamics area. The global existence or non-existence of the classical solution to an inviscid Bénard system is an open and challenging problem, even in the two-dimensional case. Therefore, it is of interest to consider the Bénard system with partial viscosity. Inspired by recent work [13, 31, 32], we first consider the global weak solution for the 2D Bénard system with partial dissipation. Secondly, we establish some regularity criteria for the corresponding system. 
Science Foundation of Qinghai Province (2017-ZJ-902); Open Research Fund Program of State key Laboratory of Hydroscience and Engineering (No. sklhse-2017-A-05); The Science and Technology Major Project of Qinghai Province Natural Science Foundation (No. 2015-SF-A4-3).

Funding

Not applicable.

List of abbreviations

We denote by $L^{p}=L^{p}\left(\mathbb{R}^{2}\right)$ the usual Lebesgue space, and by $H^{n}=\left\{u \in L^{2}\left(\mathbb{R}^{2}\right) \mid \nabla^{n} u \in L^{2}\right\}$ the usual Sobolev space.

Availability of data and materials

Not applicable.

Competing interests

The authors declare that they have no competing interests.

\section{Authors' contributions}

LM conceived and designed the work. LM drafted the manuscript. LM revised the manuscript. LM and LZ read and approved the final version.

\section{Author details}

${ }^{1}$ Department of Mathematics and Computer, Panzhihua University, Panzhihua, P.R. China. ${ }^{2}$ College of Computer Science, Sichuan University, Chengdu, P.R. China. ${ }^{3}$ State Key Laboratory of Plateau Ecology and Agriculture, Department of Computer Technology and Applications, Qinghai University, Xining, P.R. China. ${ }^{4}$ Information Management Center, Sichuan University, Chengdu, P.R. China.

\section{Publisher's Note}

Springer Nature remains neutral with regard to jurisdictional claims in published maps and institutional affiliations.

Received: 1 November 2017 Accepted: 25 April 2018 Published online: 22 May 2018

\section{References}

1. Cao, C., Wu, J.: Global regularity for the $2 \mathrm{D}$ MHD equations with mixed partial dissipation and magnetic diffusion. Adv Math. 226, 1803-1822 (2011)

2. Ambrosetti, A., Prodi, G.: A Primer of Nonlinear Analysis. Cambridge Studies in Advanced Mathematics. Cambridge University Press, Cambridge (1995)

3. Chandrasekhar, S.: Hydrodynamic and Hydromagnetic Stability. Courier Corporation, New York (2013)

4. Farhat, A., Jolly, M., Titi, E.: Continuous data assimilation for the $2 \mathrm{D}$ Bénard convection through velocity measurements alone. Physica D 303, 59-66 (2015)

5. Foias, C., Manley, O., Temam, R.: Attractors for the Bénard problem: existence and physical bounds on their fractional dimension. Nonlinear Anal. 11, 939-967 (1987)

6. Rabinowitz, P.H.: Existence and nonuniqueness of rectangular solutions of the Bénard problem. Arch. Ration. Mech. Anal. 29, 32-57 (1968)

7. Ma, T., Wang, S.: Rayleigh-Bénard convection: dynamics and structure in the physical space. Commun. Math. Sci. 5, 553-574 (2007)

8. Neustupa, J., Siginer, D.: Existence and structure of steady solutions of the Bénard problem in a two dimensional quadrangular cavity. Nonlinear Anal. 123/124, 68-88 (2015)

9. Palese, L.: On the stability of the rotating Bénard problem. Ann. Acad. Rom. Sci. Ser. Math. Appl. 6, 4-20 (2014)

10. Anh, C.T., Son, D.T.: Pullback attractors for nonautonomous 2D Bénard problem in some unbounded domains. Math. Methods Appl. Sci. 36, 1664-1684 (2013)

11. Wu, G., Xue, L.: Global well-posedness for the 2 D inviscid Bénard system with fractional diffusivity and Yudovich's type data. J. Differ. Equ. 253, 100-125 (2012)

12. Ye, Z.: Regularity criterion of the 2D Bénard equations with critical and supercritical dissipation. Nonlinear Anal. 156, 111-143 (2017)

13. Cheng, J., Du, L.: On two-dimensional magnetic Bénard problem with mixed partial viscosity. J. Math. Fluid Mech. 17, 769-797 (2015)

14. Zhou, Y., Fan, J., Nakamura, G.: Global Cauchy problem for a 2D magnetic Bénard problem with zero thermal conductivity. Appl. Math. Lett. 26, 627-630 (2013)

15. Ma, L.: Global regularity results for the $2 \frac{1}{2} \mathrm{D}$ magnetic Bénard system with mixed partial viscosity. Appl. Anal. (2018). https://doi.org/10.1080/00036811.2017.141603

16. Ma, L.: Global existence of smooth solutions for three-dimensional magnetic Bénard system with mixed partial dissipation, magnetic diffusion and thermal diffusivity. J. Math. Anal. Appl. 461, 1639-1652 (2018)

17. Zhang, Z., Tang, T.: Global regularity for a special family of axisymmetric solutions to the three dimensional magnetic Bénard problem. Appl. Anal. (2017). https://doi.org/10.1080/00036811.2017.1376661

18. Lemarié-Rieusset, P.G.: Recent Developments in the Navier-Stokes Problem. Chapman \& Hall/CRC Research Notes in Mathematics. Chapman \& Hall/CRC, Boca Raton (2002)

19. Sather, J.O.: The initial-boundary value problem for the Navier-Stokes equations in regions with moving boundaries. Ph.D. thesis, University of Minnesota (1963)

20. Serrin, J.: The initial value problem for the Navier-Stokes equations. In: Nonlinear Problems, pp. 69-98. University of Wisconsin Press, Madison (1963) 
21. Temam, R.: Navier-Stokes Equations. Theory and Numerical Analysis. Studies in Mathematics and Its Applications. North-Holland, Amsterdam (1977)

22. Zhang, Z: Serrin-type regularity criterion for the Navier-Stokes equations involving one velocity and one vorticity component. Czechoslov. Math. J. 68, 219-225 (2018)

23. Zhang, Z., Yang, X.: Navier-Stokes equations with vorticity in Besov spaces of negative regular indices. J. Math. Anal. Appl. 440, 415-419 (2016)

24. Zhang, Z., Zhong, D., Huang, X.: A refined regularity criterion for the Navier-Stokes equations involving one non-diagonal entry of the velocity gradient. J. Math. Anal. Appl. 453, 1145-1150 (2017)

25. Cavalcanti, M., Domingos Cavalcanti, V., Lasiecka, I., Webler, C.: Intrinsic decay rates for the energy of a nonlinear viscoelastic equation modeling the vibrations of thin rods with variable density. Adv. Nonlinear Anal. 6, 121-145 (2017)

26. Goubet, O., Hamraoui, E.: Blow-up of solutions to cubic nonlinear Schrödinger equations with defect: the radial case. Adv. Nonlinear Anal. 6, 183-197 (2017)

27. Morales, F., Naranjo Álvarez, S.: The interaction between PDE and graphs in multiscale modeling. Opusc. Math. 37, 327-345 (2017)

28. Ma, L.: Global existence of three-dimensional incompressible magneto-micropolar system with mixed partial dissipation, magnetic diffusion and angular viscosity. Comput. Math. Appl. 75, 170-186 (2018)

29. Ma, L.: On two-dimensional incompressible magneto-micropolar system with mixed partial viscosity. Nonlinear Anal., Real World Appl. 40, 95-129 (2018)

30. Regmi, D:: Global weak solutions for the two-dimensional magnetohydrodynamic equations with partial dissipation and diffusion. Nonlinear Anal. 144, 157-164 (2016)

31. Cheng, J., Li, S.: Global weak solutions and regularity for two-dimensional Boussinesq equations with partial dissipation and thermal diffusivity. J. Math. Anal. Appl. 428, 794-803 (2015)

32. Chen, M.: Global well-posedness of the $2 \mathrm{D}$ incompressible micropolar fluid flows with partial viscosity and angular viscosity. Acta Math. Sci. 33(4), 929-935 (2013)

33. $Y u, H .:$ Global regularity to the $2 \mathrm{D}$ incompressible MHD with mixed partial dissipation and magnetic diffusion in a bounded domain. Acta Math. Sci. Ser. B Engl. Ed. 37, 395-404 (2017)

34. Fan, J., Alzahrani, F.S., Hayat, T., Nakamura, G., Zhou, Y.: Global regularity for the 2D liquid crystal model with mixed partial viscosity. Anal. Appl. 13, 185-200 (2015)

35. Fan, J., Malaikah, H., Monaquel, S., Nakamura, G., Zhou, Y.: Global Cauchy problem of 2D generalized MHD equations. Monatshefte Math. 175, 127-131 (2014)

36. Cao, C., Wu, J.: Global regularity for the two-dimensional anisotropic Boussinesq equations with vertical dissipation. Arch. Ration. Mech. Anal. 208, 985-1004 (2013)

37. Cao, C., Regmi, D., Wu, J.: The 2D MHD equations with horizontal dissipation and horizontal magnetic diffusion J. Differ. Equ. 254, 2661-2681 (2013)

\section{Submit your manuscript to a SpringerOpen ${ }^{\circ}$ journal and benefit from:}

- Convenient online submission

- Rigorous peer review

- Open access: articles freely available online

- High visibility within the field

- Retaining the copyright to your article

Submit your next manuscript at $\boldsymbol{~ s p r i n g e r o p e n . c o m ~}$ 